\title{
Synthesis of Antenna Arrays and Parasitic Antenna Arrays with Mutual Couplings
}

\author{
M. Thevenot, C. Menudier, A. El Sayed Ahmad, G. Zakka El Nashef, F. Fezai, Y. Abdallah, \\ E. Arnaud, F. Torres, and T. Monediere
}

XLIM, OSA, UMR CNRS 6172, University of Limoges, 123 Avenue Albert Thomas, 87060 Limoges Cedex, France

Correspondence should be addressed to C. Menudier, cyrille.menudier@xlim.fr

Received 1 June 2011; Revised 17 October 2011; Accepted 31 October 2011

Academic Editor: Hoi Shun Lui

Copyright (C) 2012 M. Thevenot et al. This is an open access article distributed under the Creative Commons Attribution License, which permits unrestricted use, distribution, and reproduction in any medium, provided the original work is properly cited.

\begin{abstract}
A synthesis method to design multielement antennas with couplings is presented. The main objective is to perform a rigorous determination of the electromagnetic characteristics involved in the design, especially with arrays of moderate sizes. The aim is to conceive jointly and efficiently the antenna and the circuits to connect (feed distribution network, power amplifiers, reactive loads, etc.). The subsequent objective is to improve the understanding and capabilities of strongly coupled antennas. As a whole, the synthesis procedure is then applied to different antenna architectures in order to show its efficiency and versatility. A focus on some antenna concepts where the management of couplings is a key factor to improve the performances is presented. After describing the synthesis procedure, the first category of coupled multielement antenna studied concerns radiating arrays in linear or circular polarization. A design including couplings effects on an active array is also presented. Then, the method is applied to parasitic antenna arrays and a specific investigation on reflectarray antenna is performed as they can be considered as a particular case of parasitic arrays.
\end{abstract}

\section{Introduction}

1.1. Context. Multielement antennas are widely used for years in wireless communications because of their potentialities in terms of high gain beam scanning and complex beam shaping. Most of applications of radiating arrays concerns very large panel (more than 20 wavelength side) with several hundreds or thousands of elements because they are often dedicated to specific applications in the field of space or military missions. The high number of elements allows a high beam scan resolution for radar [1] or a well define contour to optimize (equivalent isotropic radiated power) EIRP for beam shaping [2].

Nevertheless, needs for moderate-size multielement antennas are growing because of the fast evolution of consumer telecommunication market. To obtain high data rate, an antenna gain increase is required. Moreover, as the devices including radiating elements must face a constant service evolution, multielement antennas with reconfiguration capabilities and moderate gain (between 10-20 dB for a directive pattern) would be of great interest to perpetuate telecommunications infrastructure for both end-users and operators.

Whatever the multielement antenna size, one of the main difficulties to tackle consists in reaching the highest efficiency for the design and avoiding some phenomena like scan blindness, especially while couplings are strongly impacting the performances. Furthermore, the electromagnetic performance optimization is a critical point to reduce the antenna cost, especially in the case of consumer applications.

Therefore, accurate modeling methods are representing a key factor for a successful design. Nevertheless, the electromagnetic size of the multielement antenna (in terms of wavelengths) and the accuracy required to satisfactorily represent small geometric details lead to large calculation volume, and thus, to prohibitive execution time. That is why several modeling methods have been developed.

1.2. Summary of Modeling Methods. Several approaches have been investigated for radiating arrays, and especially for large arrays. The easiest way to determine the radiation pattern 
consists in the product of an isolated element and an array factor [3]. However, it neglects both couplings and edge effects. A better and widely used approach is based on the calculation of an active element pattern (AEP), which takes into account a particular state of coupling [4]. It corresponds to the behavior of a neighbored element when the others are terminated in matched loads. The radiation pattern of the whole array is then calculated with the array factor and this active pattern, assumed to be same whatever the element location on the panel. Even if it does not account for edge effects and different coupling states, the results are efficient provided the array is large enough. In order to overcome these edge effects, improvements have been performed by considering an original induced element pattern Method (OIEPM) which consists in considering an interior element group and two edge element groups to constitute a subarray [5]. Thanks to this approach, both edge effects and a better approximation of couplings are accounted provided that the number of elements contained in the edge element group is enough and has been well defined. In fact, the authors have shown that this choice has a strong impact on results.

As they directly impact the performances of the antennas in multibeam telecommunications, direct radiating arrays (DRAs), radar, or in direction-of-arrival (DOA) estimations, mutual couplings have been widely studied in the literature. Apart from the above methods, several formalisms directly refer to mutual couplings and array synthesis. Circuit theory from [6] has been extensively used and allows estimating the effects of couplings with a relative easiness. More recently, detailed studies dedicated to mutual coupling of antenna arrays operating in transmitting and receiving modes have been presented [7-9]. The authors consider the antenna array in receiving mode with an external plane wave excitation. Moreover, the antenna elements are terminated with known impedances contrary to [6] where open-circuit terminations are considered in transmitting mode. This method, known as receiving-mutual-impedancemethod (RMIM), has proven a good efficiency for mutual coupling compensation in DOA estimation. A recent review of other methods is also given in [10].

Other formalisms have been developed to design large array. The Floquet modal analysis is a very fast way to determine the radiation pattern of a neighbored element considered in an infinite and periodic lattice [11]. However, it supposes that couplings are identical whatever the radiating element location, that is, that they are identical. The edge effects are also neglected. Some extensions of this formalism have been presented in [12] to extract an active element pattern and the array scattering matrix.

A subsequent problematic in the antenna array modeling consists in an accurate determination of fine details, especially when complex radiating elements (aperture-coupled patches, high impedance surfaces) are considered. In order to model the different details, some methods have been developed. Among them, the scale-changing technique (SCT) consists in a successive modeling of the array elements, from the smallest to the highest [13]. The different sub-domains are cascaded through scale changing networks, representing the electromagnetic coupling between adjacent scale levels. Such a method has been applied to the design of frequency selective surface (FSS) or reflectarrays [14]. Another approach based on a decomposition of the computation domain related to the electromagnetic fields is also presented in [15]. It allows a fast modeling of large array. These two methods present the advantage to avoid hybridization between simulation tools which can be a source of inaccuracy when joining formulations. The main difficulty remains the splitting of the different scale or volume which strongly affects the results and require expert users.

Through the different methods aforementioned, it appears that the main difficulty for an antenna designer is to choose the method with the best approximation, depending on the complexity of the radiating element and the size of the array.

1.3. Motivations for the Proposed Approach. The previous approaches have essentially been justified because research and engineering on multielement antennas are based on very wide panel containing several hundreds or thousands of elements. Moreover, a full-wave simulation of such meshing volume would have been unfeasible until a recent past. In fact, apart from supercomputers, desktop computers or workstations have now reached very large computing capabilities, especially thanks to the strong increase of memory sizes on 64 bits systems and multicore support. Moreover, an important development of fast multipole methods (FMMs) has allowed a faster resolution of antenna problems with iterative solvers based on Method of Moments (MoM) [16, 17]. Commercial software, like FEKO, includes a multilevel FMM to simulate large electromagnetism structure with a user-friendly interface [18]. However, the user must be aware that such methods, even if they are based on full-wave formalism, require some care while considering convergence criterion.

Another critical point that must be noticed is the optimization through synthesis procedures. As a parametric analysis is more and more time consuming when the electromagnetic characteristics are well detailed, a synthesis procedure is necessary to accurately define the exact weightings satisfying an objective pattern or polarization goal.

In fact, in the case of moderate-size arrays (below several hundreds of elements), a part of the previous methods leads to inefficient synthesis results. Formalisms based on Floquet modal decomposition do not represent the finiteness of the structure whereas edge effects become higher. Moreover, in case of different radiating element nature or aperiodic lattice, the results will be inaccurate. The same conclusions are valid for the AEP method. In the case of OIEPM, SCT, or MLFMM, more versatile structures can be modeled with a quite good approximation of couplings and edge effects. However, the result accuracy is strongly dependent of the user skills and experience with these methods. Therefore, it seems to be difficult to design such moderate-size array without the support of a full-wave method combined to an analytical rigorous formalism for speed improvement. 
In this article, we focus on a simple and versatile method accounting for couplings and easy to integrate in a synthesis process. It is partially based on previous results on strongly coupled feeds in electromagnetic bandgap (EBG) [19]. By combining full-wave modeling to compute each elementary cell radiation patterns and the array scattering matrix, a synthesis procedure is used to compute rigorous excitation weightings of the array. Added to its accuracy, the originality of this approach consists in the fact that it is multipurpose and not only dedicated to antenna array. In fact, it can also be used to synthesize reactive loads of a reconfigurable array and it can be easily suited to parasitic antenna array (PAA) and to reflectarrays, which can be considered as a specific case of a PAA. In these last two cases, the exact determination of mutual coupling is a crucial point, especially if a reconfigurable architecture is investigated.

The next section describes the method and the synthesis procedure including couplings. In Section 3, it is applied to antenna arrays with different radiation objectives in linear or circular polarization. An example with an active array is also given to use the method for predicting power amplifier performances while they are loaded on active impedances (due to couplings). Measurements are given to validate the synthesis procedure. In Section 4, the method is arranged to be used with PAA and reflectarrays.

\section{Theoretical Formalism}

The coupling between two antennas close from each other can occur because of radiation in free space, propagation of surface waves, capacitive and inductive couplings, and so forth. In the design of antenna arrays, the coupling phenomena should not be neglected. Indeed, the diagrams of the sources in an array are generally different from the isolated element pattern. In addition, the mutual coupling affects the antenna input impedance, that is, a part of the energy radiated by each port is captured by the nearby accesses. If these energies are not dissipated by isolators, they can interfere with the feed network (for a passive antenna) or the LNAs (for an active antenna) which are connected to the antenna ports. Consequently, it affects the radiation pattern and may damage the efficiency.

2.1. Coupling Mechanisms and Input Impedances. The mutual coupling between antennas induces electrical perturbations. Let us consider a distribution of antennas " $i$ " excited by feeds characterized by their input impedances $Z_{G i}$ and the electromotive force (emf) $E_{g i}$. This hypothesis is illustrated in Figure 1. $Z_{E i}$ are the resultant impedances of the antennas. These impedances take into account the mutual coupling between the antennas. The radiation pattern $\Phi$ of the array is a linear combination of the elementary radiation patterns $\Phi_{i}$ of each elementary antenna. An elementary radiation pattern (or AEP) is obtained while exciting only the " $i$ " antenna with a normalized power, while the other antennas " $j \neq i$ " are connected to a port impedance $Z_{G j}$. $\Phi_{i}$ contains the unnormalized radiated fields.

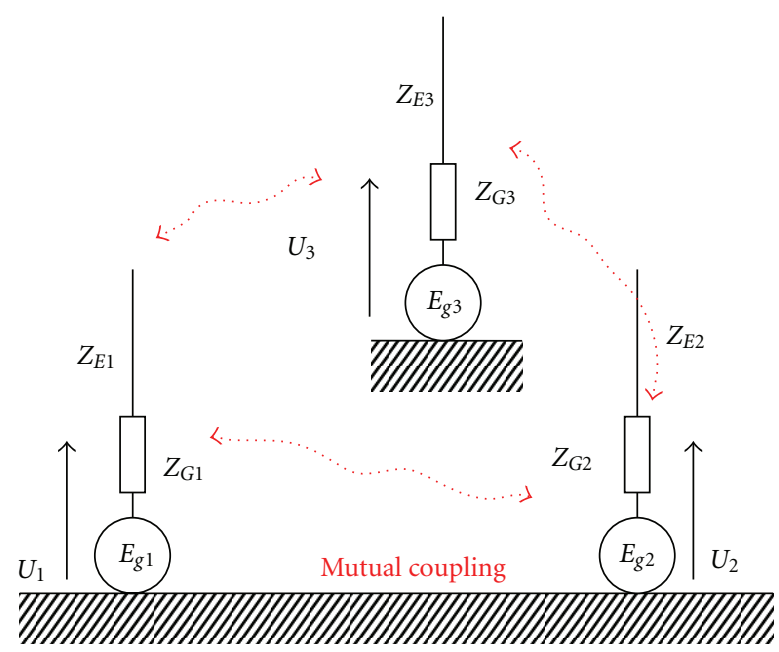

Figure 1: Illustration of couplings between antennas.

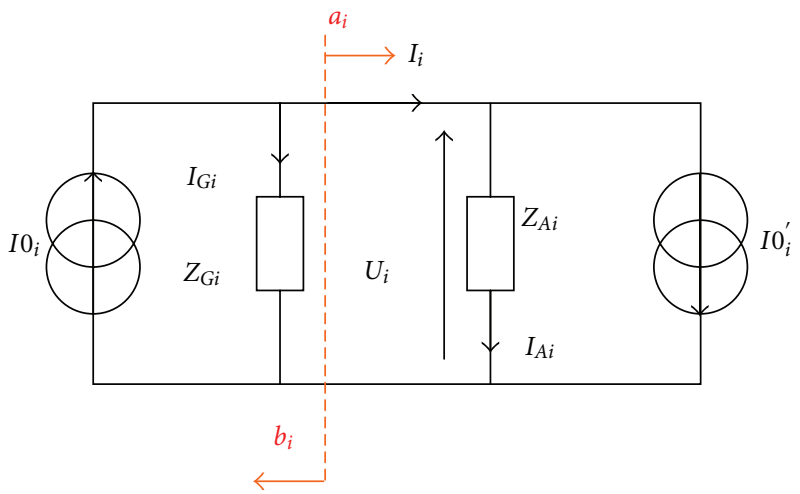

FIGURE 2: Equivalent electrical scheme of an antenna in the array.

The resulting radiation pattern results from (1):

$$
\left[\begin{array}{llll}
\Phi_{1} & \Phi_{2} & \cdots & \Phi_{n}
\end{array}\right] \cdot\left(\begin{array}{c}
\beta_{1} \\
\beta_{2} \\
\vdots \\
\beta_{n}
\end{array}\right)=\Phi .
$$

The relation linking the weights $\left\{\beta_{1}, \beta_{2}, \beta_{3}, \ldots, \beta_{n}\right\}$ with the voltages $\left\{U_{1}, U_{2}, U_{3}, \ldots, U_{n}\right\}$ applied to each antenna port will be given later.

In order to help the understanding, the equivalent electrical scheme of Figure 2 is proposed [19]. It is valid for each elementary antenna in the array. This scheme considers that all the antennas are fed. $I O_{i}$ are the feeding currents $\left(I 0_{i}=\right.$ $\left.E_{g i} / Z_{G i}\right) . I 0_{i}^{\prime}$ are the currents induced by the radiations of the other antennas. These currents are driven by the voltages that are applied on each elementary antenna of the array. They are expressed by (2):

$$
I 0_{i}^{\prime}=\sum_{j \neq i} \alpha_{i j} U_{j}
$$

$\left[\alpha_{i j}\right]$ is the coupling admittance matrix. 
$Z_{A i}$ are the intrinsic impedances of the elementary antennas. They can be deduced while $I 0_{i}^{\prime}$ are off, that is, when all the other antennas $j \neq i$ were short circuited.

With reference to Figure 2, (3) can be easily deduced,

$$
U_{i}=\frac{Z_{A i} Z_{G i}}{Z_{A i}+Z_{G i}}\left(I 0_{i}-\sum_{j \neq i} \alpha_{i j} U_{j}\right),
$$

leading to the matrix relation of (4):

$$
\begin{gathered}
\left(\begin{array}{cccc}
\frac{Z_{A 1}+Z_{G 1}}{Z_{A 1} Z_{G 1}} & \alpha_{12} & \cdots & \alpha_{1 n} \\
\alpha_{21} & \frac{Z_{A 2}+Z_{G 2}}{Z_{A 2} Z_{G 2}} & \cdots & \alpha_{2 n} \\
\cdots & \cdots & \cdots & \cdots \\
\alpha_{n 1} & \alpha_{n 2} & \cdots & \frac{Z_{A n}+Z_{G n}}{Z_{A n} Z_{G n}}
\end{array}\right)\left(\begin{array}{c}
U_{1} \\
U_{2} \\
\vdots \\
U_{n}
\end{array}\right) \\
=\left(\begin{array}{c}
I 0_{1} \\
I 0_{2} \\
\vdots \\
I 0_{n}
\end{array}\right) .
\end{gathered}
$$

Notice that the driven voltages $\left(U_{1}\right.$ to $\left.U_{n}\right)$ depend on the feeding ports $\left\{I 0_{i} ; Z_{G i}\right\}$ and the interactions $\left[\alpha_{i j}\right]$ of the other antennas in the array.

The input impedance $Z_{E i}$, defined by (5) for each elementary antenna is obtained while all the antennas are simultaneously fed with the compliant weight. From (5) and the current law applied to Figure 2, $Z_{E i}$ can be written as in (6) for the coupled array:

$$
\begin{gathered}
Z_{E i}=\frac{U_{i}}{I_{i}}, \\
Z_{E i}=\frac{Z_{G i} U_{i}}{Z_{G i} I 0_{i}-U_{i}} .
\end{gathered}
$$

This result exhibits that the input impedance appears as a function of both the mutual couplings and the driven voltages.

The next paragraph deals with the impedance matching of this multiport array antenna.

2.2. Impedance Matching. The objective is to achieve the impedance matching of all access, that is, to change the characteristic impedance of each antenna port $\left(Z_{G i}\right)$ by a load impedance $Z_{L i}$ satisfying (7) and (8):

$$
\begin{gathered}
Z_{L i} \approx Z_{E i}^{*}, \\
Z_{E i} \approx Z_{L i} \cdot \exp ^{-j .2 . \angle Z_{L i}} .
\end{gathered}
$$

The feeding currents can be deduced from (6) and (8):

$$
I 0_{i} \approx U_{i} \cdot \frac{1+\exp ^{+j .2 . \angle Z_{L i}}}{Z_{L i}} .
$$

Inserting (9) into (4) leads to the relation (10):

$$
\begin{gathered}
\left(\begin{array}{cccc}
\frac{Z_{L 1}^{*}-Z_{A 1}}{Z_{A 1} Z_{L 1}^{*}} & \alpha_{12} & \cdots & \alpha_{1 n} \\
\alpha_{21} & \frac{Z_{L 2}^{*}-Z_{A 2}}{Z_{A 2} Z_{L 2}^{*}} & \cdots & \alpha_{2 n} \\
\cdots & \cdots & \cdots & \cdots \\
\alpha_{n 1} & \alpha_{n 2} & \cdots & \frac{Z_{L_{n}}^{*}-Z_{A n}}{Z_{A n} Z_{L_{n}}^{*}}
\end{array}\right)\left(\begin{array}{c}
U_{1} \\
U_{2} \\
\vdots \\
U_{n}
\end{array}\right) \\
=\left(\begin{array}{c}
0 \\
0 \\
\vdots \\
0
\end{array}\right) .
\end{gathered}
$$

Then, the aim is to seek $Z_{L i}^{*}$ values that will satisfy the relation (10) and thus realize the overall matching of the array. The $U_{i}$ data are linked to the radiation pattern objectives.

2.3. Solving the Problem. The last equations describe the behavior of the array while all the antennas are fed and loaded by the relevant impedances.

The resolution of these equations requires the coupling admittance matrix $\left[\alpha_{i j}\right]$ characterizing the array. The scattering matrix is the more efficient way to extract these unknowns of the multiport problem. The next paragraph leads to a formalism of the problem using the scattering matrix.

Let us consider that only the antenna " $i$ " is fed while the others ( $" j ", j \neq i$ ) are loaded by impedances $Z_{G j}$. Figure 3 illustrates the electrical scheme involving two antennas among the array while only " $i$ " is fed. fed.

$I_{i i}$ is the current in the port of the antenna " $i$ " while " $i$ " is

$I_{j i}$ is the current in the port of the antenna " $j$ " $(j \neq i)$ while " $i$ " is fed.

$U_{j i}$ is the voltage in the port of the antenna " $j$ " while only " $i$ " is fed...

Using Figure 3, relations (11) can be written:

$$
\begin{gathered}
I_{i, i}=\frac{U_{i, i}}{Z_{A i}}+\sum_{j \neq i} \alpha_{i, j} U_{j, i}, \\
I_{j, i}=\frac{U_{j, i}}{Z_{A j}}+\sum_{k \neq j} \alpha_{j, k} U_{k, i} .
\end{gathered}
$$

The two previous relations meet the following simplified expressions:

$$
\begin{aligned}
I_{i, i} & =\sum_{j} \alpha_{i, j} U_{j, i}, \\
I_{j, i} & =\sum_{k} \alpha_{j, k} U_{k, i}
\end{aligned}
$$

with $\alpha_{i, i}=1 / Z_{A i}$ and $\alpha_{j, j}=1 / Z_{A j}$. 


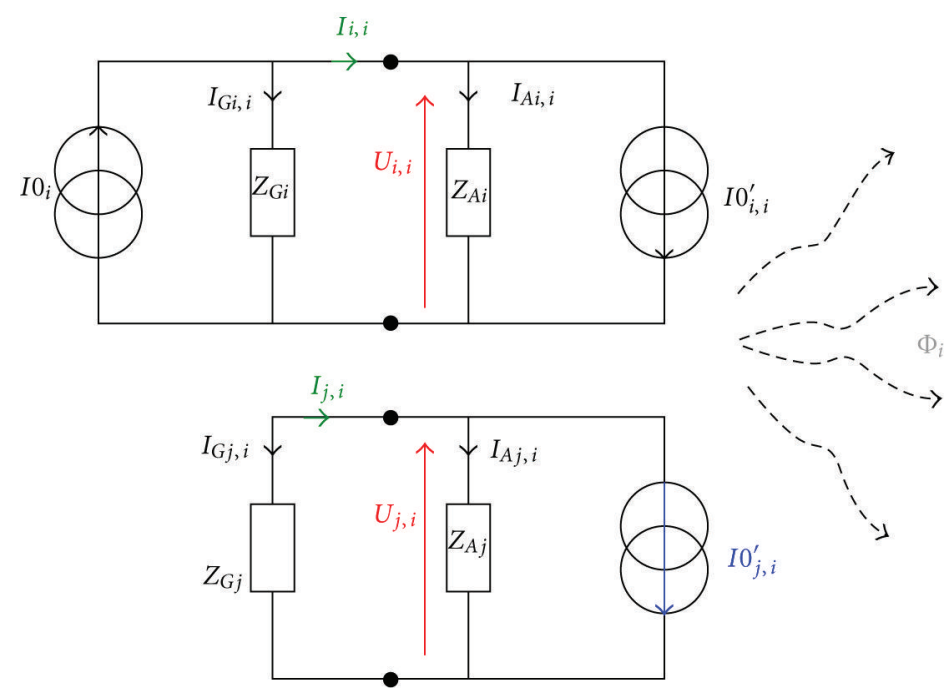

FIgURE 3: Equivalent electrical scheme of one antenna fed $(i)$ with another loaded with the impedance $Z_{G j}$.

Finally, the currents that are coupled in the antenna ports while only " $i$ " is fed are given by the relation (13):

$$
\left(\begin{array}{c}
I_{1, i} \\
I_{2, i} \\
\vdots \\
I_{n, i}
\end{array}\right)=\left[\begin{array}{cccc}
\alpha_{1,1} & \alpha_{1,2} & \cdots & \alpha_{1, n} \\
\alpha_{2,1} & \alpha_{2,2} & \cdots & \alpha_{2, n} \\
\cdots & \cdots & \cdots & \cdots \\
\alpha_{n, 1} & \alpha_{n, 2} & \cdots & \alpha_{n, n}
\end{array}\right] \cdot\left(\begin{array}{c}
U_{1, i} \\
U_{2, i} \\
\vdots \\
U_{n, i}
\end{array}\right)
$$

If we now consider that all the port can be fed simultaneously, the relation (14) is defined to take into account the part of each excitation:

$$
\begin{aligned}
\left(\begin{array}{llll}
I_{1,1} & I_{1,2} & \cdots & I_{1, n} \\
I_{2,1} & I_{2,2} & \cdots & I_{2, n} \\
\cdots & \cdots & \cdots & \cdots \\
I_{n, 1} & I_{n, 2} & \cdots & I_{n, n}
\end{array}\right) \\
=\left[\begin{array}{llll}
\alpha_{1,1} & \alpha_{1,2} & \cdots & \alpha_{1, n} \\
\alpha_{2,1} & \alpha_{2,2} & \cdots & \alpha_{2, n} \\
\cdots & \cdots & \cdots & \cdots \\
\alpha_{n, 1} & \alpha_{n, 2} & \cdots & \alpha_{n, n}
\end{array}\right] \cdot\left(\begin{array}{llll}
U_{1,1} & U_{1,2} & \cdots & U_{1, n} \\
U_{2,1} & U_{2,2} & \cdots & U_{2, n} \\
\cdots & \cdots & \cdots & \cdots \\
U_{n, 1} & U_{n, 2} & \cdots & U_{n, n}
\end{array}\right) .
\end{aligned}
$$
(14):

The admittance matrix of (15) is then deduced from

$$
\begin{aligned}
& {\left[\begin{array}{llll}
\alpha_{1,1} & \alpha_{1,2} & \cdots & \alpha_{1, n} \\
\alpha_{2,1} & \alpha_{2,2} & \cdots & \alpha_{2, n} \\
\cdots & \cdots & \cdots & \cdots \\
\alpha_{n, 1} & \alpha_{n, 2} & \cdots & \alpha_{n, n}
\end{array}\right]} \\
& =\left(\begin{array}{llll}
I_{1,1} & I_{1,2} & \cdots & I_{1, n} \\
I_{2,1} & I_{2,2} & \cdots & I_{2, n} \\
\cdots & \cdots & \cdots & \cdots \\
I_{n, 1} & I_{n, 2} & \cdots & I_{n, n}
\end{array}\right) \cdot\left(\begin{array}{llll}
U_{1,1} & U_{1,2} & \cdots & U_{1, n} \\
U_{2,1} & U_{2,2} & \cdots & U_{2, n} \\
\cdots & \cdots & \cdots & \cdots \\
U_{n, 1} & U_{n, 2} & \cdots & U_{n, n}
\end{array}\right)^{-1},
\end{aligned}
$$

with

$$
\alpha_{i, i}=\frac{1}{Z_{A i}} .
$$

Let us remind the scattering matrix formalism. The normalized power waves are defined versus the voltage and the current in the ports of impedance $Z_{G i}$ :

$$
a_{i}=\frac{U_{i}+Z_{G i} \cdot I_{i}}{2 \cdot \sqrt{R_{G i}}}, \quad b_{i}=\frac{U_{i}-Z_{G i}^{*} \cdot I_{i}}{2 \cdot \sqrt{R_{G i}}} .
$$

The scattering matrix $[S]$ links the input waves $\left(a_{i}\right)$ to the reflected ones $\left(b_{i}\right)$ :

$$
(b)=[S] \cdot(a) .
$$

Using this formalism, the expression of the voltages and the currents in the ports is given by (19) and (20):

$$
U_{i j}=\left(\frac{Z_{G_{i}}^{*}}{\sqrt{R_{G_{i}}}} \cdot \delta_{i j}+\frac{Z_{G_{i}}}{\sqrt{R_{G_{i}}}} \cdot S_{i j}\right) \cdot a_{j},
$$




$$
I_{i j}=\left(\delta_{i j}-S_{i j}\right) \cdot \frac{a_{j}}{\sqrt{R_{G_{i}}}}
$$

with

$$
\delta_{i j}=\mid \begin{array}{ll}
1 & \text { if } i=j \\
0 & \text { if } i \neq j
\end{array}
$$

The admittance matrix in (15) can be expressed thanks to the scattering matrix of the array antenna. It is defined by the relation (22). In this expression, we remain that $Z_{G i}$ are the characteristic impedances of the ports that are used to normalize the $S$ parameters:

$$
[\alpha]=\frac{\operatorname{diag}\left(\frac{1}{\sqrt{R_{G}}}\right) \cdot([I]-[S])}{\operatorname{diag}\left(\frac{Z_{G}^{*}}{\sqrt{R_{G}}}\right)+\operatorname{diag}\left(\frac{Z_{G}}{\sqrt{R_{G}}}\right) *[S]} .
$$

It has been previously written in (1) that the radiation pattern is synthesized with a linear combination of the diagrams $\Phi_{1 \ldots n}$. These ones result from the successive excitation of the $n$ antennas with the same incident power. Then, the complex coefficients $\left\{\beta_{1}, \beta_{2}, \beta_{3}, \ldots, \beta_{n}\right\}$ allow to calculate the voltages and the currents injected into each antenna. Equations (23) remind these relations deduced from (19) and (20):

$$
\begin{gathered}
(U)=\left[\operatorname{diag}\left(\frac{Z_{G}^{*}}{\sqrt{R_{G}}}\right)+\operatorname{diag}\left(\frac{Z_{G}}{\sqrt{R_{G}}}\right) *[S]\right] \cdot(\beta), \\
(I)=\operatorname{diag}\left(\frac{1}{\sqrt{R_{G}}}\right) \cdot([I]-[S]) \cdot(\beta) .
\end{gathered}
$$

The input impedance $Z_{E}$ of the coupled system is then deduced from (23) and (24):

$$
Z_{E}=\frac{\left[\operatorname{diag}\left(Z_{G}^{*} / \sqrt{R_{G}}\right)+\operatorname{diag}\left(Z_{G} / \sqrt{R_{G}}\right) *[S]\right] \cdot(\beta)}{\operatorname{diag}\left(1 / \sqrt{R_{G}}\right) \cdot([I]-[S]) \cdot(\beta)} .
$$

At last, to satisfy the impedance matching of the array, the antennas must be fed with the waves $a$ given by the relation (25) through ports having the characteristic impedances that are the conjugates of (25), that is, $Z_{L i}=Z_{E i}^{*}$ :

$$
a=\operatorname{diag}\left(\frac{\sqrt{R_{L}}}{Z_{L}^{*}}\right) \cdot\left[\operatorname{diag}\left(\frac{Z_{G}^{*}}{\sqrt{R_{G}}}\right)+\operatorname{diag}\left(\frac{Z_{G}}{\sqrt{R_{G}}}\right) *[S]\right] \cdot(\beta) .
$$

Once the theoretical formalism has been described, it can then be applied to solve a multielement antenna problem through the following guidelines.

At first, a full-wave simulation of the array is required in order to extract the $[S]$ matrix and each active element radiation pattern $\phi_{i}$ of the array. Notice that the load impedance on each antenna termination is known at this step.

Then, these data are provided to MATLAB in order to apply the formalism previously detailed. An objective

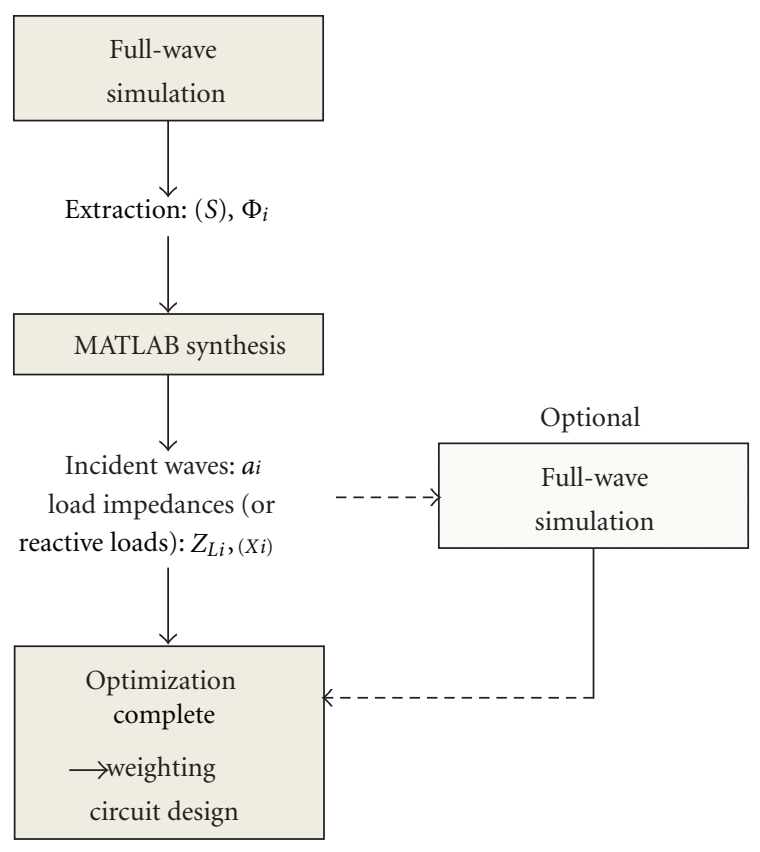

FIgURE 4: Synthesis approach summarized.

radiation pattern $\phi$ is provided to the synthesis procedure in order to define the best weights $\beta_{i}$ according to (1). Following the different developments of part 2, the input impedances $Z_{E i}$ of the coupled system are calculated with (25) and the final weightings $a_{i}$ are deduced with (25).

Now, the designer knows the best-fit values for $a_{i}$ and $Z_{E i}$, that is, $Z_{L i}$ which are conjugate. Notice that a fullwave simulation can be performed at this step by changing the excitation weightings in the simulation software, for example, CST MWS, by the given dataset. This step remains optional as it will be shown in the application examples because the excitation weightings found with the synthesis process are accurately defined. At last, it is then possible to design the feed network allowing the provided excitation weightings. Notice that in the case in the array, we recommend a final cosimulation of the feed distribution network and the antenna array to evaluate the influence of the radiating elements on the feed lines. In fact, the feed network lines can be coupled with waves of the radiating elements and it can affect the final weightings. In the applications presented in this article, we have chosen designs with feed networks located on the rear side of the antenna to minimize this effect. Therefore, a cosimulation of the array and its feed network is performed only to validate the synthesis procedure and there is no need to adjust the weightings after the array synthesis. These different steps are summarized on Figure 4.

In terms of computation time, the only time-consuming step is the first one because of the extraction of the $[S]$ matrix and active radiation patterns $\phi_{i}$ by a full-wave simulation. However, we aim at designing moderate-size array and/or parasitic arrays with moderate sizes as it was described in the Introduction. Therefore, with a reasonable workstation, the time spread is between some minutes and a few hours. Then, 


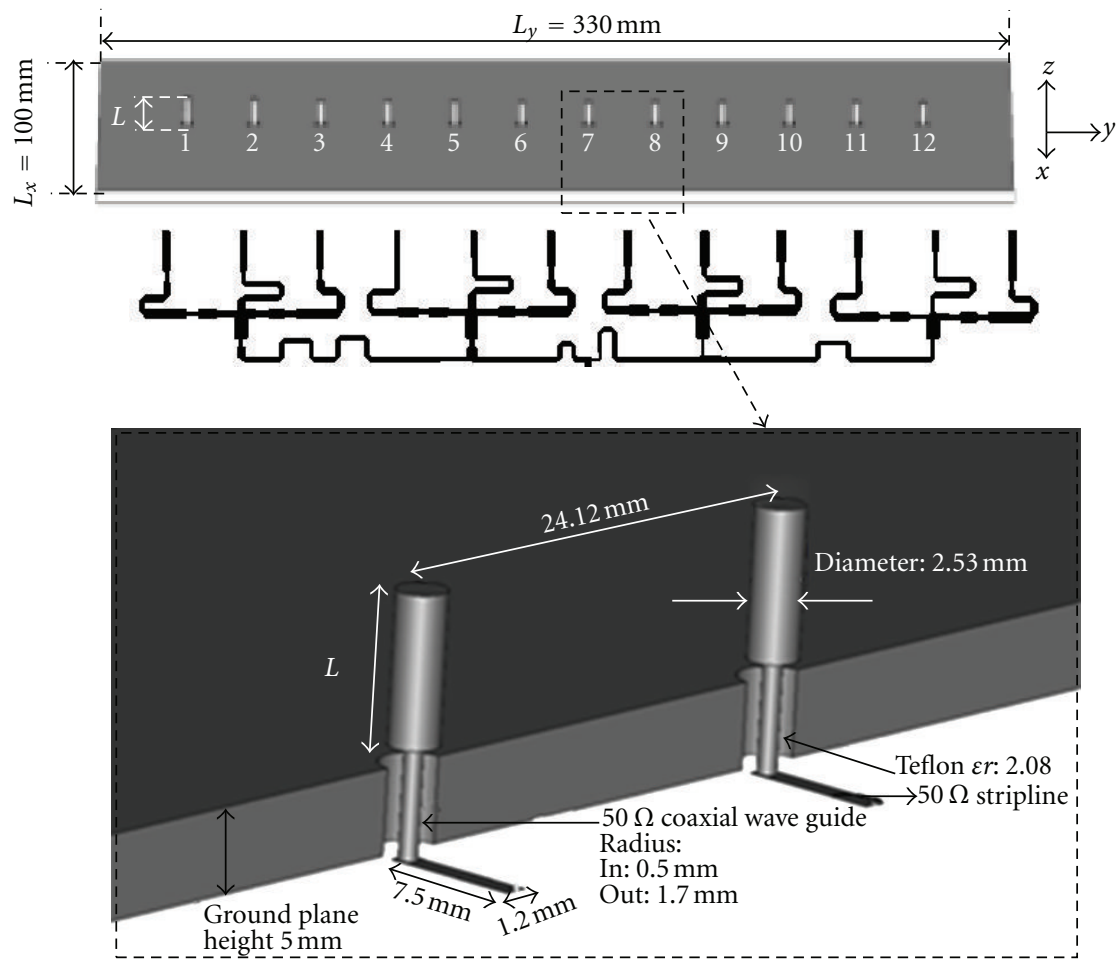

Figure 5: Array of 12 monopoles with its feed distribution network.

the MATLAB synthesis is reduced to a few seconds to find the best weightings. That is why this approach can be considered as efficient without too much time consuming. Notice that there is no approximation for the scattering matrix and for the different active radiation pattern computation with this approach.

In terms of radiation objective, this paper focuses only on the synthesis of pattern in one direction, especially in the array alignment. Beam shaping can also be applied with the approach described, but it will only be added in our future works. For readers interested in beam shaping, [20] presents a synthesis method with mutual couplings and an interesting overview of the other methods is presented in their introduction.

\section{Synthesis of Antenna Arrays}

As the different steps to define the most appropriate weights to satisfy the radiation objective in a coupled multielement antenna have been described, this section is dedicated to three cases of application with measurements.

\subsection{Monopole Linear Array}

3.1.1. Antenna Description. As a first example of application, a monopole array for the HyperLAN2 bandwidth (5.47$5.72 \mathrm{GHz}$ ) is presented. The synthesis objective is to obtain a directive beam in the array alignment $(x-y$ plane on Figure 3 ). Therefore, the couplings must be taken into account to avoid important radiation pattern deteriorations and to maximize the directivity in this direction.

As depicted on Figure 5, the antenna is composed of 12 elements of $2.53 \mathrm{~mm}$ diameter with $0.45 \lambda_{0}(24.12 \mathrm{~mm})$ spacing.

These ones are fasten to a ground plane whose dimensions are $L x=100 \mathrm{~mm}$ and $L y=330 \mathrm{~mm}$. The feed network is printed on the bottom face of a Duroid 6002 substrate and it is connected to the monopoles through the ground plane. The substrate has a 2.94 relative permittivity and a 0.0012 loss tangent.

3.1.2. Synthesis Description and Results. In order to synthesize the right weightings for the feed distribution network, the method described in Section 2 is applied and the design can be summarized in three steps.

At first, a full-wave simulation of the monopole array is performed with CST MWS to obtain the global scattering matrix $[S]$ and the radiation patterns $\Phi_{i}$. This study shows that the couplings between monopoles could reach $-12 \mathrm{~dB}$ in the worst cases. In Table 1 , the complex weights $\beta_{i}$ are then deduced according to (1) and the radiation objective $(\Phi)$.

Then, the optimum weights $a_{i}$ and the impedances $Z_{L i}$ which simultaneously satisfy the objective radiation pattern and the matching of all the feeding ports are calculated using (25) and (25). The corresponding values are given in Table 1 with the optimized monopole lengths. Notice that these lengths have been defined to suit both the different impedance values resulting from the synthesis procedure and a minimization of the feed distribution network complexity. 


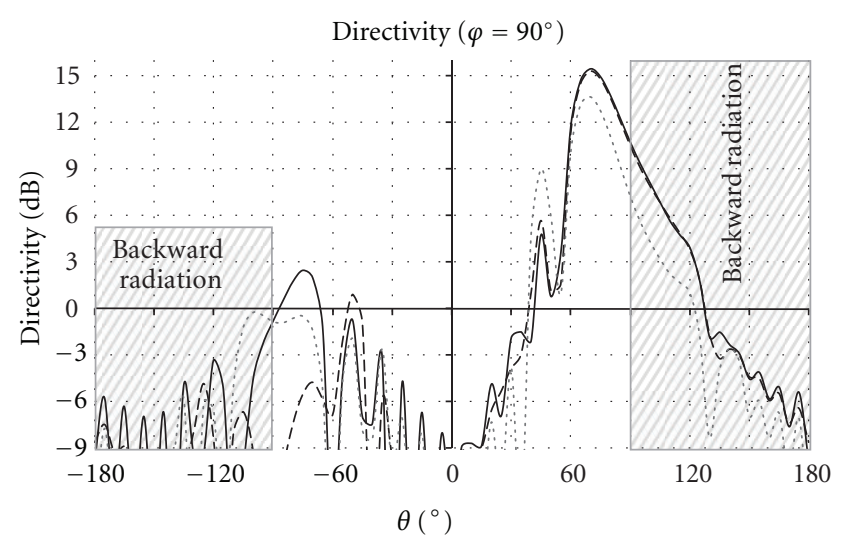

_ Linear combination of the element pattern (equation (1))

--- Entire structure simulation

(feed network connected with the monopoles)

Synthesis with couplings neglected

FIgURE 6: Synthesized radiation pattern with couplings and theoretical linear combination of the monopole patterns (objective) at 5.60 GHz. Results with couplings neglected are also presented.

The last step consists in the feed network design to obtain the weights and the impedance matching specified in Table 1. The optimized layout is presented Figure 3. In order to validate the design, a cosimulation of the monopole antenna array connected to the Agilent Momentum feed distribution network design is performed in CST design studio. The comparison of the synthesized radiation pattern obtained by this simulation is compared to the theoretical linear combination on Figure 6. The gain and dielectric losses are evaluated in Table 2.

In order to show the interest of including couplings into the synthesis procedure, a comparison of the results has been made with a classical design where they are neglected. Neglecting couplings correspond to a simplified $[S]$ matrix (quoted $S^{\prime \prime}$ ) expressed by (26):

$$
\begin{array}{cc}
S_{i j}^{\prime \prime}=S_{i j} & \text { for } i=j, \\
S_{i j}^{\prime \prime}=0 & \text { for } i \neq j .
\end{array}
$$

The new values for $Z_{L i}$ and $a_{i}$ are determined using (14) and (15) while $\beta_{i}$ stays unchanged. Another feed network has been designed to comply with the objectives $a_{i}$. The effect on the radiation pattern appears on Figure 4 where a $2 \mathrm{~dB}$ directivity decrease is observed at $5.6 \mathrm{GHz}$. A more detailed presentation is available in [21].

3.1.3. Measurements. The monopole array and the feed distribution network were manufactured as can be seen on Figure 7. The feed network is glued at the rear of the ground plane. Notice that screws were added to secure the RF contacts but their interactions with the circuit have been checked and considered as insignificant. An SMA connector is used to feed the array.

The antenna return loss is represented in Figure 8 and shows a matching better than $-15 \mathrm{~dB}$ on the radiation

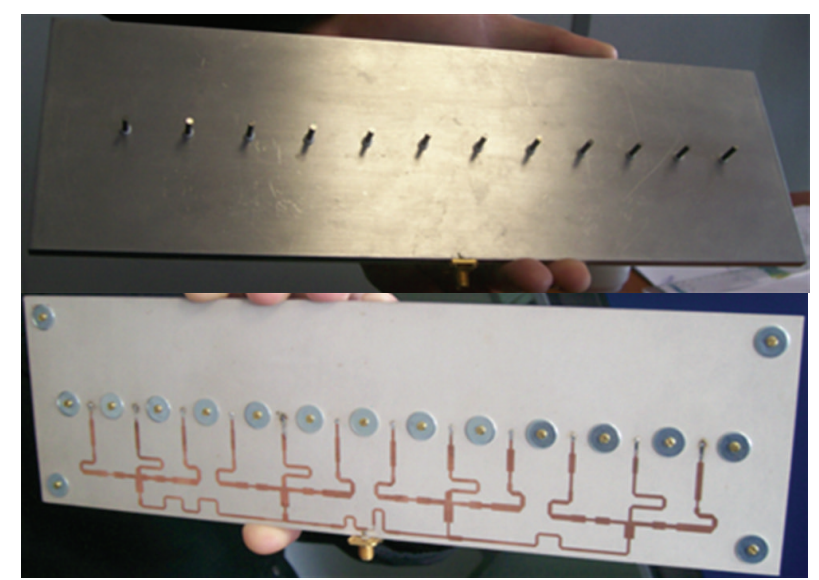

FIGURE 7: Top view of the manufactured prototype and back view with the feed distribution network.

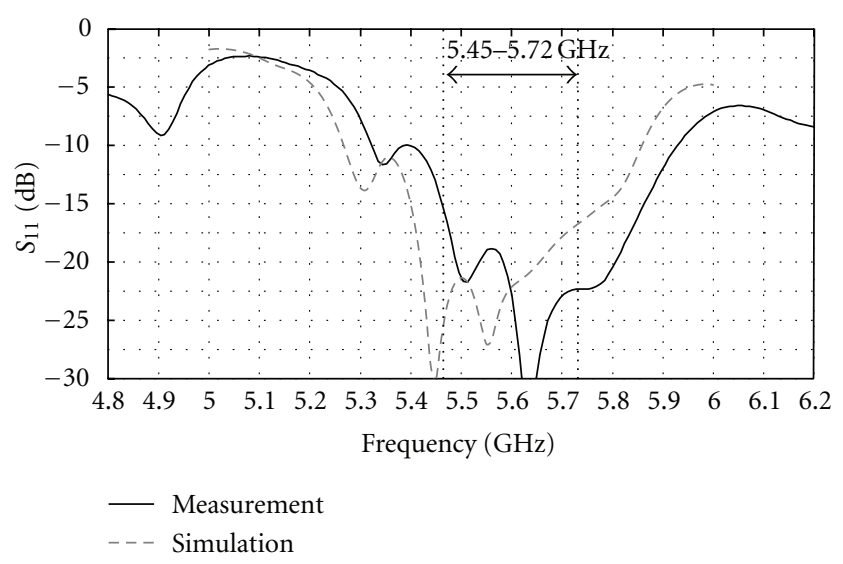

FIGURE 8: $\mathrm{S}_{11}$ comparison between simulation and manufactured prototype.

bandwidth. A slight discrepancy of $50 \mathrm{MHz}$ can be observed compared to the simulation but it represents only a $0.9 \%$ of frequency shift that can be due to the mesh accuracy during simulation or manufacture tolerance. Notice that the simulation results correspond to the feed network design (with Agilent Momentum) connected to the monopole array in CST design studio. Several resonances appear in the bandwidth due to the dispersive behavior of the array scattering matrix on the one hand and of the feed network on the other hand.

The radiation patterns are compared in Figure 9 in the $y-z$ plane at $5.6 \mathrm{GHz}$. A good agreement with the predicted pattern is observed. The difference between the simulated and the measured gain over the frequency range is lower than $0.5 \mathrm{~dB}$. This difference might be caused by metallic losses in the feed network and the measurement accuracy of the anechoic chamber $( \pm 0.5 \mathrm{~dB})$.

These results validate the synthesis method of Section 2 because a significant improvement has been observed in measurement and a $2 \mathrm{~dB}$ increase of directivity is obtained compared to a design where the couplings are neglected. 
TABLE 1: Weightings applied to the monopole array and resulting antenna impedances.

\begin{tabular}{|c|c|c|c|c|}
\hline \multirow[t]{2}{*}{ Monopoles $i$} & \multirow[t]{2}{*}{ Length (mm) } & \multirow[t]{2}{*}{$\beta_{i}$ (=weights for the coupled radiation patterns) } & \multicolumn{2}{|c|}{$\begin{array}{c}\text { Normalized incident waves }\left(a_{i}\right) \text { and load } \\
\text { impedances }\left(Z_{L i}\right) \text { that optimize the efficiency } \\
\text { (couplings included) }\end{array}$} \\
\hline & & & $a_{i}$ & $Z_{L i}$ \\
\hline 1 & 10.8 & $0.286 \cdot \exp ^{\left(-j * 48^{\circ}\right)}$ & $0.288 \cdot \exp ^{\left(-j * 49^{\circ}\right)}$ & $29+j * 13$ \\
\hline 2 & 9.8 & $0.293 \cdot \exp ^{\left(j * 155^{\circ}\right)}$ & $0.30 \cdot \exp ^{\left(j * 152^{\circ}\right)}$ & $32+j * 10$ \\
\hline 3 & 9.3 & $0.279 \cdot \exp ^{\left(-j * 3^{\circ}\right)}$ & $0.286 \cdot \exp ^{\left(-j * 6^{\circ}\right)}$ & $31+j * 9$ \\
\hline 4 & 9.3 & $0.284 \cdot \exp ^{\left(-j * 155^{\circ}\right)}$ & $0.291 \cdot \exp ^{\left(-j * 164^{\circ}\right)}$ & $30+j * 1$ \\
\hline 5 & 9.3 & $0.284 \cdot \exp ^{\left(j * 47^{\circ}\right)}$ & $0.287 \cdot \exp ^{\left(j * 35^{\circ}\right)}$ & $27-j * 2$ \\
\hline 6 & 8.8 & $0.280 \cdot \exp ^{\left(-j * 120^{\circ}\right)}$ & $0.283 \cdot \exp ^{\left(-j * 124^{\circ}\right)}$ & $29+j * 7.5$ \\
\hline 7 & 8.8 & $0.266 \cdot \exp ^{\left(j * 82^{\circ}\right)}$ & $0.262 \cdot \exp ^{\left(j * 79^{\circ}\right)}$ & $24+j * 8.5$ \\
\hline 8 & 8.8 & $0.264 \cdot \exp ^{\left(-j * 74^{\circ}\right)}$ & $0.251 \cdot \exp ^{\left(-j * 77^{\circ}\right)}$ & $20+j * 8.5$ \\
\hline 9 & 8.8 & $0.254 \cdot \exp \left(j * 131^{\circ}\right)$ & $0.227 \cdot \exp ^{\left(j * 124^{\circ}\right)}$ & $15+j * 3$ \\
\hline 10 & 8.8 & $0.275 \cdot \exp \left(-j * 24^{\circ}\right)$ & $0.257 \cdot \exp ^{\left(-j * 35^{\circ}\right)}$ & $18-j * 1.5$ \\
\hline 11 & 8.8 & $0.319 \cdot \exp ^{\left(j * 180^{\circ}\right)}$ & $0.323 \cdot \exp ^{\left(j * 165^{\circ}\right)}$ & $28-j * 7$ \\
\hline 12 & 8.3 & $0.361 \cdot \exp ^{\left(j * 0^{\circ}\right)}$ & $0.377 \cdot \exp ^{\left(j * 0^{\circ}\right)}$ & $48+j * 17$ \\
\hline
\end{tabular}

TABLE 2: Simulated gain and dielectric losses.

\begin{tabular}{lcc}
\hline Frequency $(\mathrm{GHz})$ & Gain $(\mathrm{dB})$ & Dielectric losses $(\mathrm{dB})$ \\
\hline 5.47 & 14.5 & 0.77 \\
5.6 & 14.7 & 0.96 \\
5.72 & 14.6 & 0.64 \\
\hline
\end{tabular}

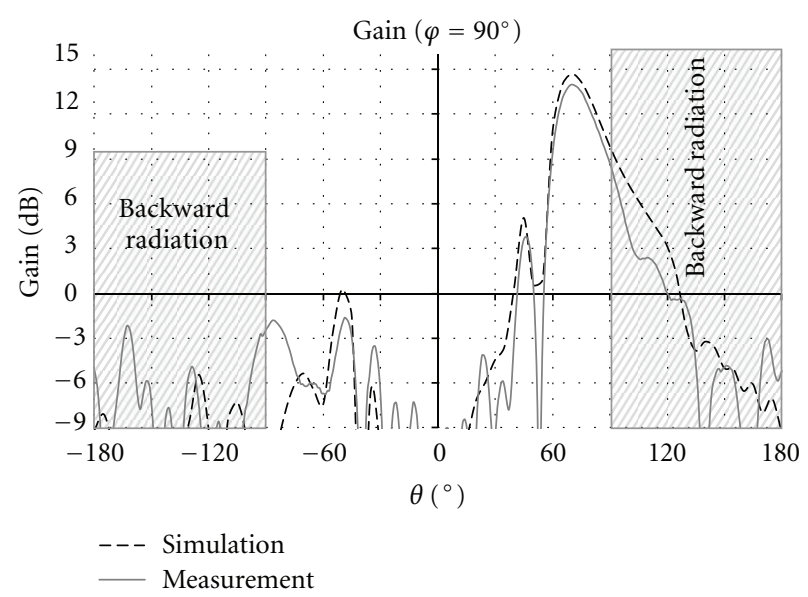

Figure 9: Comparison of the simulated radiation pattern and measurement at $f=5.6 \mathrm{GHz}$.

\subsection{Patch Array in Circular Polarization}

3.2.1. Antenna Description. In this part, the case of an antenna array in circular polarization is studied. A particular focus is made on the synthesis objective defined on an axial ratio objective and not on the radiation pattern (as in the previous part). The antenna architecture is depicted on Figure 10. It is composed of a 6 element antenna array in circular polarization and distributed on a regular hexagon lattice. An additional wire-patch antenna (WPA) is located in the center of the former hexagon for a separate link in linear

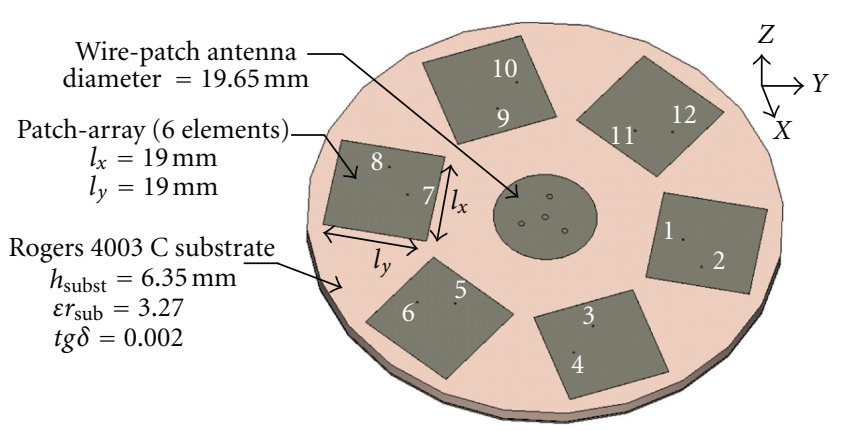

(a)

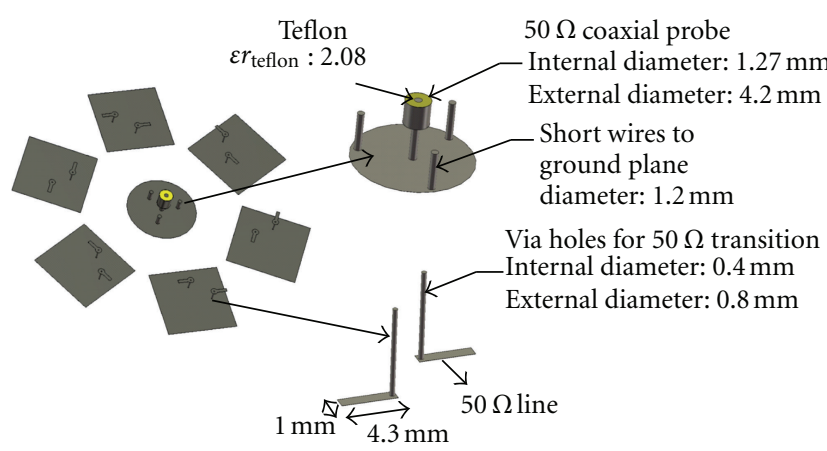

(b)

Figure 10: (a) CAD view of the circular array of patches (b) CAD view of the transitions through the feed distribution network.

polarization. The radiation bandwidth is contained between 3.4-3.55 GHz for a European Wimax application. The main difficulty to tackle in the antenna design is to generate a circular polarization with a good axial ratio whereas the patch-array elements are strongly coupled with each other and with the single wire-patch antenna. Notice that this article is focused on the patch array. The WPA design and performances will not be detailed. 


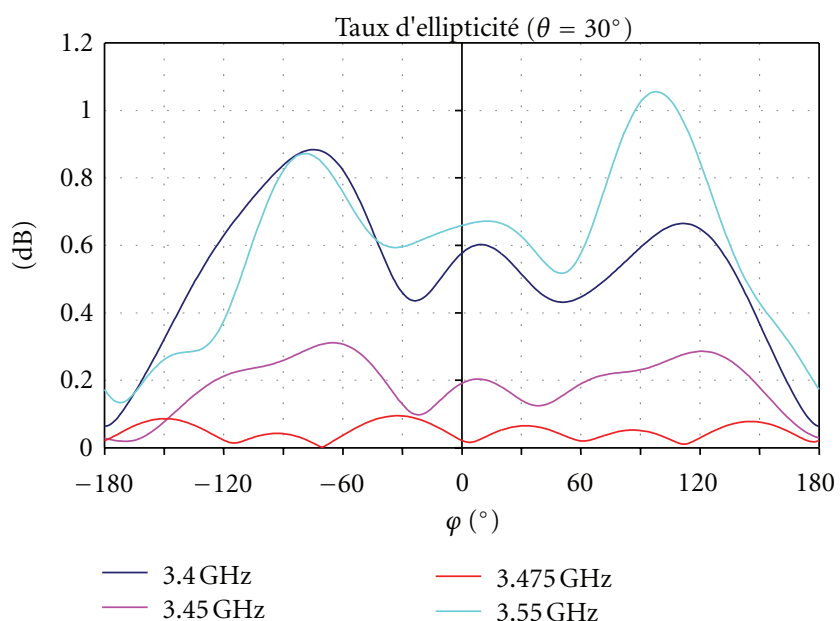

FIgURe 11: Axial ratio obtained with the linear combination of (1). Notice the agreement with the objectives at $3.5 \mathrm{GHz}$.

3.2.2. Synthesis Description and Results. In order to apply the synthesis procedure described in Section 2, the expression of (1) must be modified to include the circular polarization constraints on the radiated field components $\vec{E}_{\theta}(\theta, \varphi)$ and $\vec{E}_{\varphi}(\theta, \varphi)$.

Consider an antenna array composed of $n$ radiating elements with $x$ excitation probes on each radiator, leading to $m=x \cdot n$ probes. The multielement antenna with multiple ports is characterized by an $\left[S_{m \times m}\right]$ scattering matrix and $m$ radiation patterns obtained by successively feeding each radiator while the $(m-1)$ excitation probes are loaded on their characteristic impedances.
Thanks to this dataset, the synthesis procedure can be used by setting polarization objectives in specified directions. Each excited probe generates a radiation pattern with two components $\vec{E}_{\theta_{i}}(\theta, \varphi)$ and $\vec{E}_{\varphi_{i}}(\theta, \varphi)$.

As a consequence, the radiated polarization defined by $V_{\theta}(\theta, \varphi)$ and $V_{\varphi}(\theta, \varphi)$ in (27) and (28) can be controlled in a direction of the space with two excitation probes $i$ and $k$ :

$$
\begin{aligned}
& V_{\theta}(\theta, \varphi)=\beta_{i} \cdot E_{\theta_{i}}(\theta, \varphi)+\beta_{k} \cdot E_{\theta_{k}}(\theta, \varphi), \\
& V_{\varphi}(\theta, \varphi)=\beta_{i} \cdot E_{\varphi_{i}}(\theta, \varphi)+\beta_{k} \cdot E_{\varphi_{k}}(\theta, \varphi) .
\end{aligned}
$$

To obtain a circular polarization in a direction $\left(\theta_{0}, \varphi_{0}\right)$, the relation (29) can be written:

$$
V_{\theta}\left(\theta_{0}, \varphi_{0}\right)=e^{ \pm j \pi / 2} \cdot V_{\varphi}\left(\theta_{0}, \varphi_{0}\right),
$$

leading to (29):

$$
\begin{aligned}
& \beta_{i}\left[E_{\theta_{i}}\left(\theta_{0}, \varphi_{0}\right)-j \cdot E_{\varphi_{i}}\left(\theta_{0}, \varphi_{0}\right)\right]=\cdots \\
& \quad \cdots \beta_{k}\left[-E_{\theta_{k}}\left(\theta_{0}, \varphi_{0}\right)+j \cdot E_{\varphi_{k}}\left(\theta_{0}, \varphi_{0}\right)\right] .
\end{aligned}
$$

Therefore a radiating element with two excitation probes allows to control the radiated polarization in one direction. This conclusion can be generalized to an antenna with $m$ excitation probes to satisfy a polarization objective in $\mathrm{m} / 2$ directions.

The studied antenna is composed of $n$ radiating elements with two excitation probes $(m=2 \cdot n)$. As a whole, the following equation has to be solved:

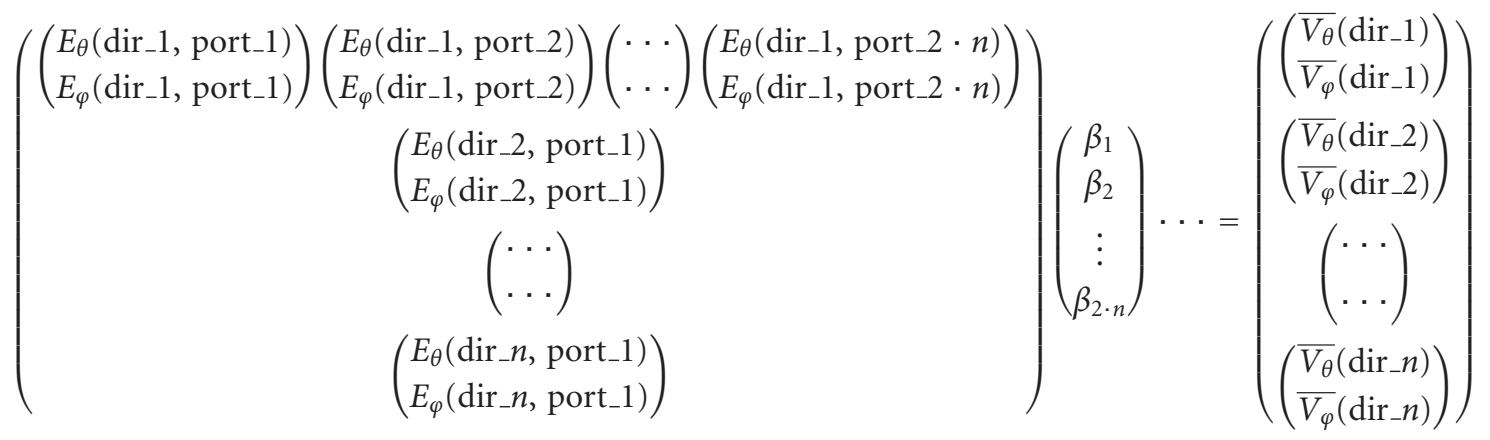

$E_{\theta}\left(\operatorname{dir} \_n\right.$, port $\left.\_m\right)$ and $E_{\varphi}\left(\right.$ port $\left.\_m\right)$ correspond to the components of the radiation pattern for each $m$ ports excited in the $n$ directions.

Once the system of (31) is defined, the synthesis procedure of Section 2 can be applied to our 6 patch array previously described.

The main lobe of the patch-array radiates in the zenith direction with a circular polarization. The whole array antenna has been optimized with the strong mutual couplings. The WPA radiates an omnidirectional pattern in linear polarization.

As before, the antenna was studied with the software CST microwave studio and the feed network has been designed with Agilent Momentum. The operating frequency is $3.475 \mathrm{GHz}$.

For each direction, the following relation between the polarization components is imposed:

$$
V_{\theta_{\text {direction_i }}}=-j \cdot V_{\varphi_{\text {direction_ }} \cdot} \text {. }
$$

Notice that the absolute phase of $V_{\theta}$ constitutes a degree of freedom to reach realistic solutions in terms of weightings. It can be expressed as (34):

$$
V_{\theta_{\text {direction. } i}}=\exp ^{j \cdot \alpha \cdot \varphi_{\text {direction_i } i}} \text { avec } \alpha \in \mathbb{Z} \text {. }
$$

The axial ratio (AR) required for the application is defined to be below $2.5 \mathrm{~dB}$ for $\theta$ varying between $-30^{\circ}$ 
TABLE 3: Results of the synthesis procedure for the 6 patch-array.

\begin{tabular}{|c|c|c|c|}
\hline \multirow[t]{2}{*}{ Excitation Probe $i$} & \multirow[t]{2}{*}{$\beta_{i}$ (=weights for the coupled radiation patterns) } & \multicolumn{2}{|c|}{$\begin{array}{l}\text { Normalized incident waves }\left(a_{i}\right) \text { and load } \\
\text { impedances }\left(Z_{L i}\right) \text { that optimize the axial } \\
\text { ratio (couplings included) }\end{array}$} \\
\hline & & $a_{i}$ & $Z_{E i}$ \\
\hline 1 & $0.388 \cdot \exp ^{\left(j * 155^{\circ}\right)}$ & $0.309 \cdot \exp ^{\left(j * 2^{\circ}\right)}$ & $41+j * 47$ \\
\hline 2 & $0.335 \cdot \exp ^{\left(-j * 90^{\circ}\right)}$ & $0.262 \cdot \exp ^{\left(j * 117^{\circ}\right)}$ & $76+j * 65$ \\
\hline 3 & $0.354 \cdot \exp ^{\left(-j * 123^{\circ}\right)}$ & $0.306 \cdot \exp ^{\left(j * 70^{\circ}\right)}$ & $37+j * 22$ \\
\hline 4 & $0.325 \cdot \exp ^{\left(-j * 19^{\circ}\right)}$ & $0.272 \cdot \exp ^{\left(j * 180^{\circ}\right)}$ & $69+j * 43$ \\
\hline 5 & $0.359 \cdot \exp ^{\left(-j * 66^{\circ}\right)}$ & $0.309 \cdot \exp ^{\left(j * 127^{\circ}\right)}$ & $35+j * 21$ \\
\hline 6 & $0.315 \cdot \exp ^{\left(j * 39^{\circ}\right)}$ & $0.267 \cdot \exp ^{\left(j * 237^{\circ}\right)}$ & $60+j * 38$ \\
\hline 7 & $0.388 \cdot \exp ^{\left(-j * 24^{\circ}\right)}$ & $0.310 \cdot \exp ^{\left(j * 182^{\circ}\right)}$ & $41+j * 47$ \\
\hline 8 & $0.335 \cdot \exp ^{\left(j * 90^{\circ}\right)}$ & $0.262 \cdot \exp ^{\left(j * 297^{\circ}\right)}$ & $77+j * 65$ \\
\hline 9 & $0.355 \cdot \exp ^{\left(j * 56^{\circ}\right)}$ & $0.306 \cdot \exp ^{\left(j * 250^{\circ}\right)}$ & $37+j * 22$ \\
\hline 10 & $0.325 \cdot \exp ^{\left(j * 161^{\circ}\right)}$ & $0.272 \cdot \exp ^{\left(j * 0^{\circ}\right)}$ & $69+j * 43$ \\
\hline 11 & $0.360 \cdot \exp ^{\left(j * 114^{\circ}\right)}$ & $0.309 \cdot \exp ^{\left(j * 307^{\circ}\right)}$ & $35+j * 21$ \\
\hline 12 & $0.314 \cdot \exp ^{\left(-j * 141^{\circ}\right)}$ & $0.266 \cdot \exp ^{\left(j * 57^{\circ}\right)}$ & $60+j * 38$ \\
\hline
\end{tabular}

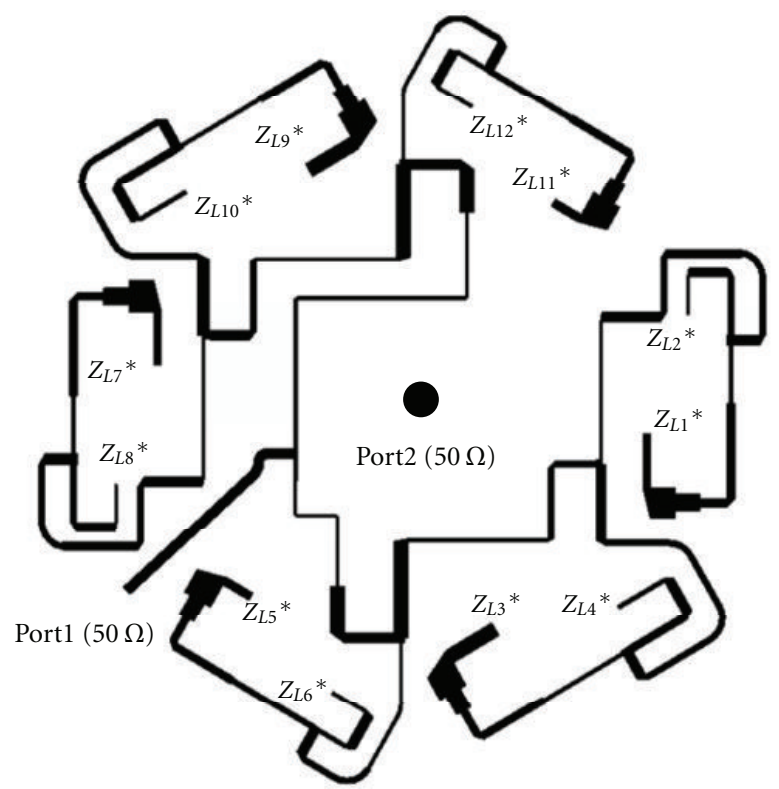

FIGURE 12: The feed network is designed to maximize the efficiency of the strongly coupled patches.

and $+30^{\circ}$, whatever the $\varphi$-plane considered. With the twelve excitation probes of the antenna, it is possible to define six polarization objectives in six directions. In our case, the objective $\mathrm{AR}$ has been set to $0 \mathrm{~dB}$ at $3.5 \mathrm{GHz}$ in the directions $\theta=30^{\circ}$ for the plane $\varphi=0,60,120,180,240$, and $300^{\circ}$. Then, the system of (31) is solved to determine the $\beta_{i}$ values. These ones, the weightings $a_{i}$, and the load impedances $Z_{L i}$ are summarized in Table 3. The resulting AR obtained in simulation with discrete ports to satisfy the weightings (without the feed distribution network) is given on Figure 11. Notice that the objective expected around 3.5 GHz agree with the simulation.

Then, the design of the microstrip feed network has been made with the Agilent Momentum software in order to perform the weights and the impedance matching specified

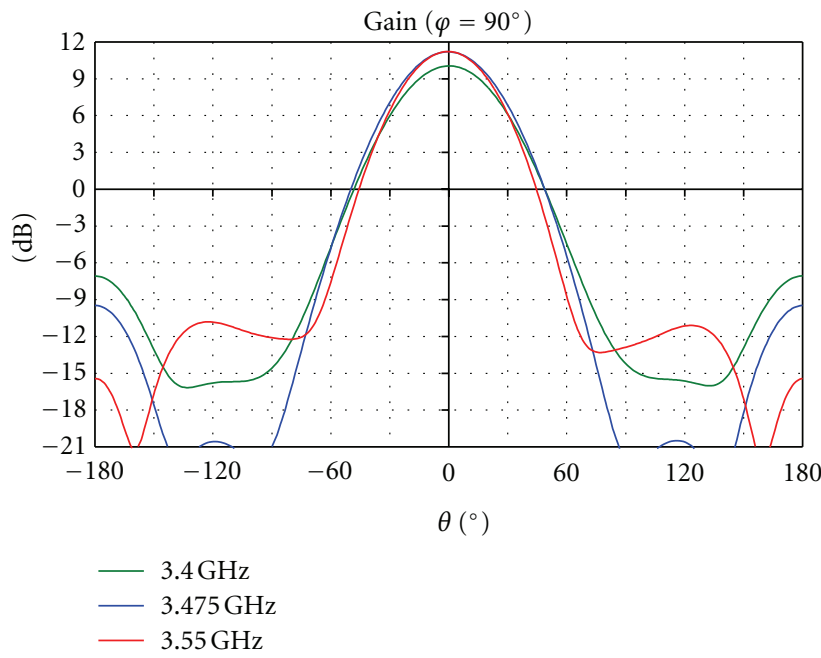

FIGURE 13: Realized gain obtained in simulation with the substrate losses.

in Table 3. The layout is shown in Figure 12. The radiation pattern obtained by a cosimulation of the feed network and the radiating elements is presented on Figure 13. A realized gain better than $10 \mathrm{~dB}$ is expected on the radiation bandwidth.

3.2.3. Measurements. The realized prototype is printed onto a $3.048 \mathrm{~mm}$ thickness Duroïd RT6002 substrate $(\varepsilon r=2.94$, $\operatorname{tg} \delta=0.0012$ ). The feed network is printed back to the antenna ground plane, onto a $0.508 \mathrm{~mm}$ thickness Duroïd 4003C substrate $(\varepsilon r=3.38, \operatorname{tg} \delta=0.0027)$. The circuit substrate is fixed to the ground plane with a glue-type PREG $4450 \mathrm{~F}(\varepsilon r=3.52$, thickness $=0.1 \mathrm{~mm}, \operatorname{tg} \delta=0.004)$. Thus, the structure consists of a circuit with two different dielectric layers separated by a ground plane. A photograph of the prototype is given in Figure 14.

The measured return loss of the antenna is compared to the cosimulation of the feed network connected to the array 


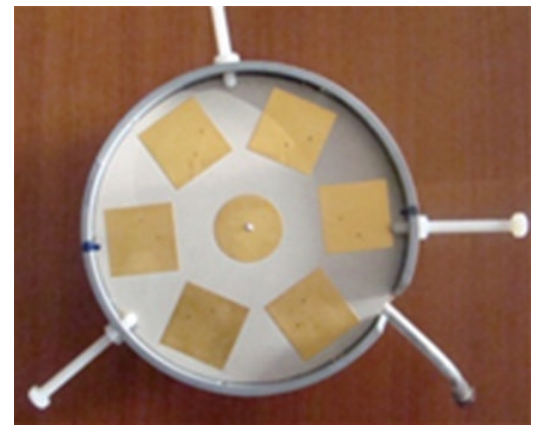

(a)

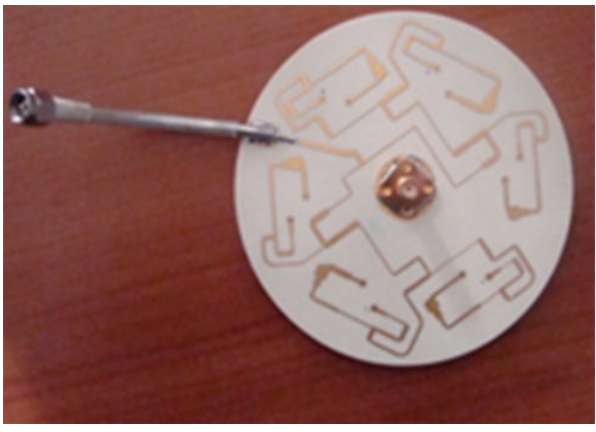

(b)

FIGURE 14: Top and back views of the manufactured prototype with the feed distribution network.

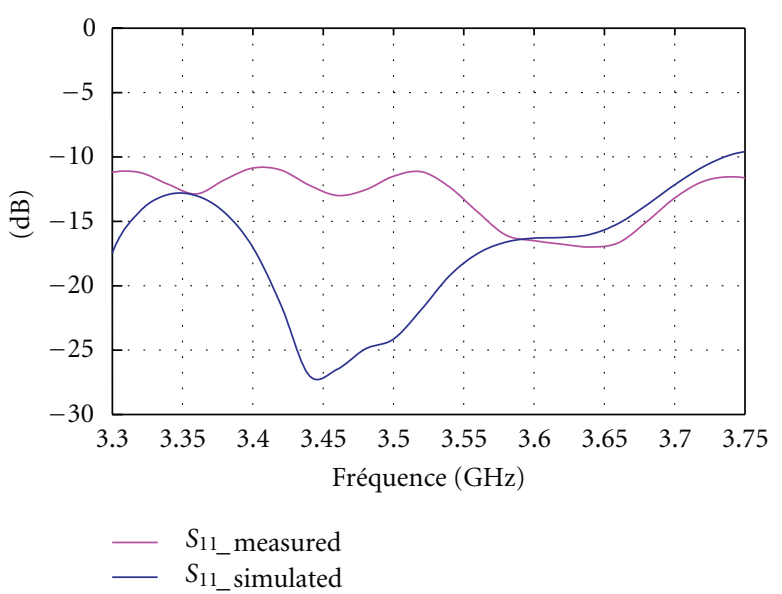

Figure 15: Comparison of return loss between cosimulation and measures.

on Figure 15. The matching is compliant with the objective between $3.4-3.55 \mathrm{GHz}$ even if the performances are lower than the prediction. This discrepancy might be caused by the complex manufacturing process and tolerances on the feed distribution network. It has been observed that the feed lines of the feed distribution network were about 50 microns below nominal values and that via holes diameter was superior to the simulation (about 10 to 20\%). Moreover, some elements like the glue thin film between the two faces of the circuit have not been simulated. At last, a lack of accuracy

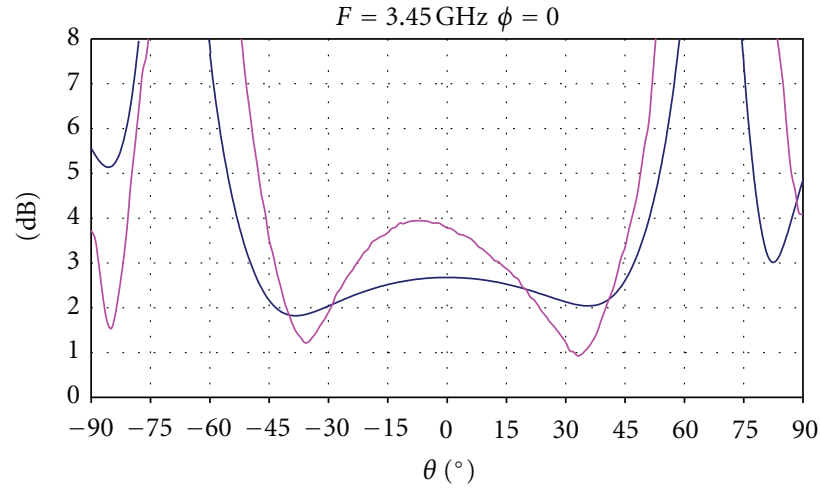

(a)

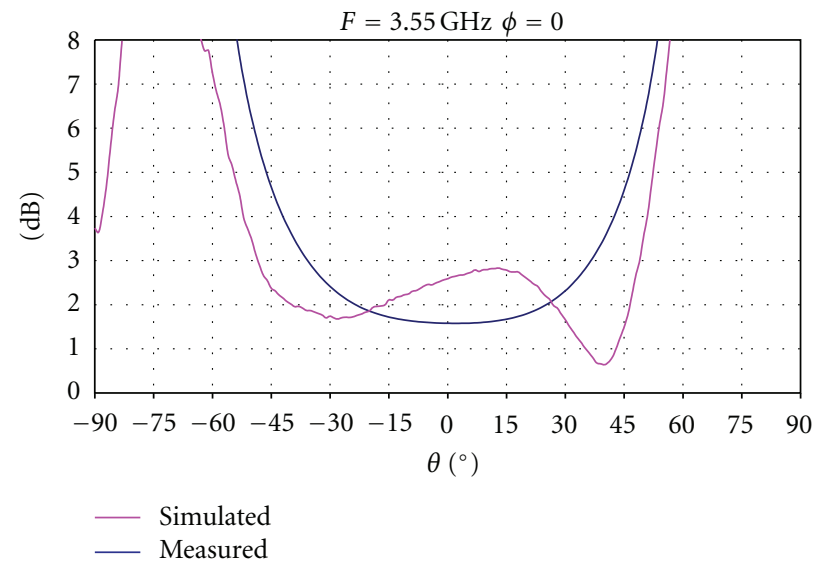

(b)

Figure 16: Comparison of $\mathrm{AR}$ at $3.45 \mathrm{GHz}$ (a) and $3.55 \mathrm{GHz}$ (b). The objective was set in the direction $\left(\theta=30^{\circ}, \varnothing=0^{\circ}\right)$.

(due to meshing) in the scattering matrix computation may also contribute to this discrepancy.

Concerning the AR, the comparison between the cosimulation with the feed distribution network and the measurement is satisfactory, especially in the directions that have been optimized $\left(\theta=30^{\circ}, \varphi=0^{\circ}\right)$. The results for the frequencies $3.45 \mathrm{GHz}$ and $3.55 \mathrm{GHz}$ are presented on Figure 16.

As confirmed by the measurement results, the synthesis procedure has been successfully applied to this antenna in circular polarization. The objectives set on the AR during the synthesis with couplings have been met. This study allows to conclude that the methodology described in Section 2 is multipurpose and can be generalized to all antenna array, whatever the radiation or polarization objective. The next part will be focused on a specific case of array with amplifiers (active array) in order to show an application of the synthesis procedure to predict performances of amplifiers.

3.3. Focus on Active Arrays. This section of the article discusses the interactions between power amplifiers (PAs) and antennas. More precisely, short relative distance between the antenna array elements may result in a high level of mutual coupling between antennas, leading to large output 


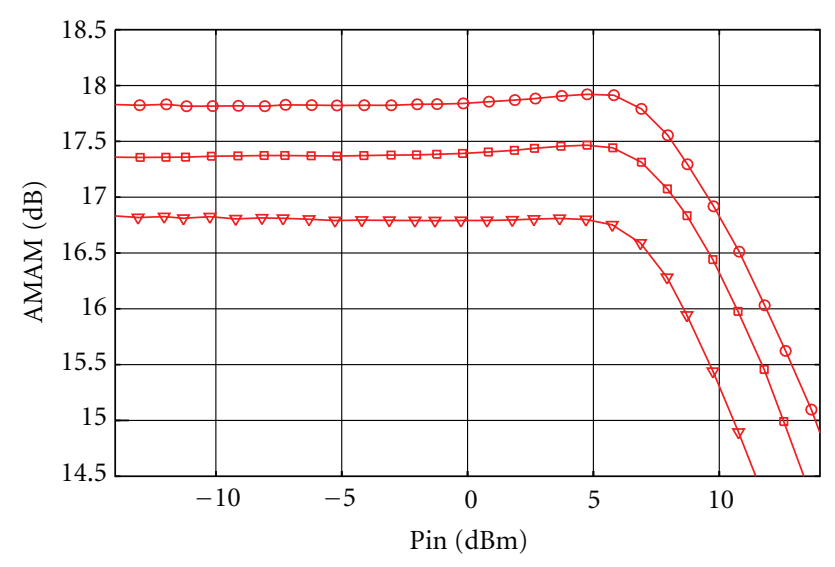

○_ Measurement@Z_load1=50ohm

$\square$ Measurement @Z_load2 $=27.2+j * 21.1 \mathrm{ohm}$

$\rightarrow$ Measurement @Z_load3 =40.3+ $j * 63.9$ ohm

Figure 17: Gain compression (AMAM) versus input power for several loading impedances $\left(Z \_l o a d 1=50 \mathrm{ohm}, \mathrm{Z} \_\right.$load $2=27.2+$ $j \cdot 21.1$, Z_load3 $=40.3+j \cdot 63.9)$.

loading mismatches (up to VSWR (voltage standing wave ratio) $=4: 1$, and more) [22]. This mismatching affects PA behavior, which in turn directly impacts the gain and phase controls of each radiating element. Thus, accurate modeling and simulation processes are needed in order to take into account the interaction between PAs and antennas correctly.

In order to show these effects, we have considered a commercial 8-14 GHz PA from NEXTEC RF (NEXTEC-RF NB00422). It exhibits a $17 \mathrm{~dB}$ small signal gain with a $27 \mathrm{dBm}$ output power at $1 \mathrm{~dB}$ gain compression and an associated power added efficiency of $17 \%$. The measurements of PA incident and reflected waves were performed using a loadpull bench, as explained in [23]. Figures 17 and 18 show, respectively, the measured compression gain (AMAM) and modulated phase (AMPM) characteristics, for three samples of load impedances for the chosen device under test (DUT).

Significant changes on PA performances over the three impedances can be noticed, showing the sensitivity of PA to its loading. These changes will affect the current distribution (e.g., magnitude and phase) applied to the antennas accesses, leading to overall performance degradation. Consequently the necessary magnitude and phase weights for the array in a given direction will be also modified once applied to PAs, degrading the array efficiency and its radiation performances.

Therefore, we developed an RF circuit behavioral model [23-25], which is a second-order extension of the so-called nonlinear scattering functions of PA. It allows taking into account large output loading impedance mismatches, that is, VSWR up to 4. The proposed model can be extracted from a simple CW load-pull measurements setup [23]. The model has been validated by comparison with measurement results obtained for several loading impedance mismatches. Those later impedances correspond to the calculated impedances obtained by the synthesis procedure as described in Section 2

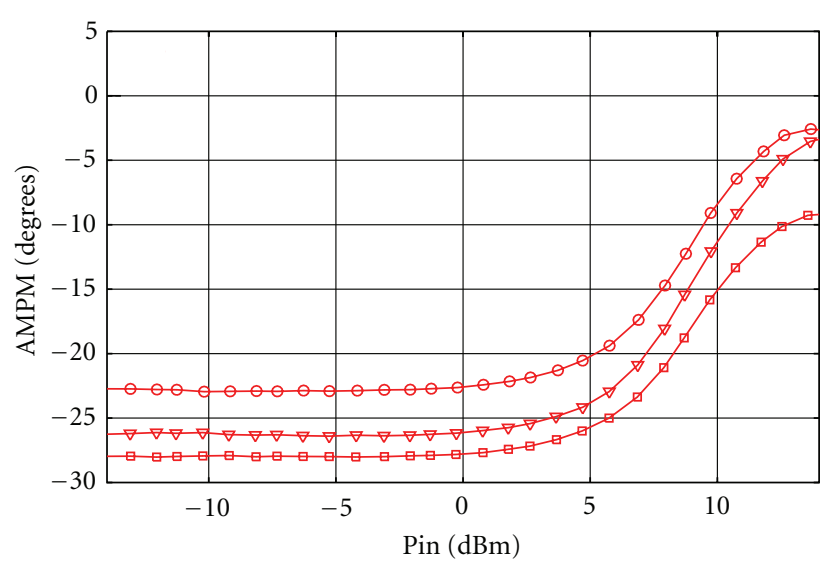

$\rightarrow-$ Measurement @Zload1 $=50 \mathrm{ohm}$
$\rightarrow-$ Measurement @ Zlload2 $=27.2+j * 21.1 \mathrm{ohm}$
$\rightarrow-$ Measurement @ Z_load3 $=40.3+j * 63.9 \mathrm{ohm}$

Figure 18: Phase modulation (AMPM) versus input power for several loading impedances $(Z$ load $1=50 \mathrm{ohms}, \mathrm{Z}$ load $2=27.2+$ $j \cdot 21.1$, Z_load3 $=40.3+j \cdot 63.9)$.

and developed in [26]. Table 4 presents an example of calculated matching impedances from the synthesis procedure for a $1 \times 4$ patch array working at $8.2 \mathrm{GHz}$ with a $0^{\circ},-10^{\circ}$, and $+15^{\circ}$ pointing angles. We should mention that interelement spacing between antennas was reduced to $0.4 \lambda_{0}$ in order to increase the mutual coupling.

It can be noticed that the optimized matching impedances of antennas are quite different from the ideal $50 \Omega$ value. This result shows the important influence of mutual coupling and the need to take this phenomenon into consideration.

The PA measurement for the incident and reflected waves was realized for some particular impedances obtained from the synthesis procedure (i.e., as in Table 4). Those impedances were defined as an output load for PA characterizing each antenna in the array. Figures 19 and 20 show the comparison between PA model and measurements in terms of gain (AMAM) and phase (AMPM) for three different antennas impedances calculated by the synthesis procedure as in Table 4.

Figures 19 and 20 show a perfect agreement. This approach clearly demonstrates the efficiency and accuracy of the second-order model, even in the presence of output loading impedance characterizing the antenna.

Thus, the knowledge of magnitude and phase variations due to PAs feeding antennas' mismatched loads allows correcting the feeding weights of the array. Therefore, the desired radiation pattern for the studied system (antennas + PAs) can be obtained. It has been experimentally validated on a $1 \times 4$ patch array working at $8.2 \mathrm{GHz}$ which has been designed using CST microwave studio.

The demonstrator included also a power splitter (1 to 4 ways), four attenuators, and four phase shifters connected to four PA, as in Figure 21. PAs are directly inserted between the phase shifters and array's antennas, the absence of isolators between PAs and antennas makes this architecture 


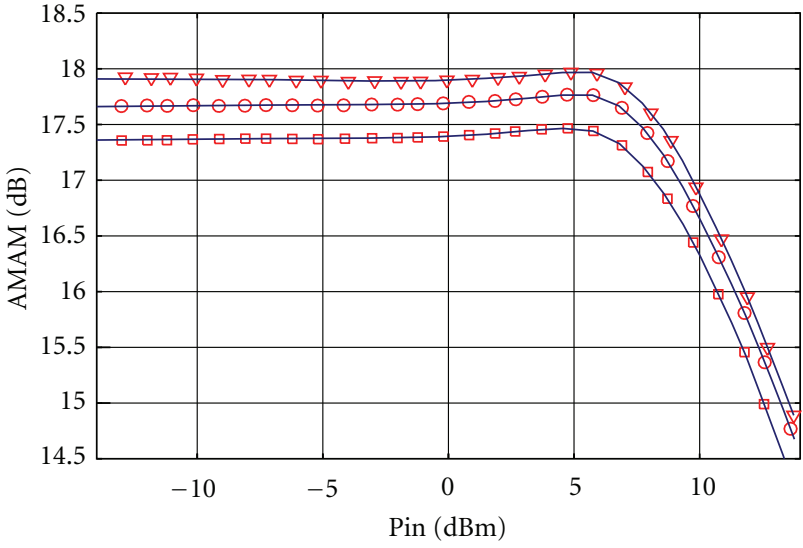

$\nabla \quad$ Measurement $@ Z \_$ant $1=57.1+j * 20.1$

○ Measurement @ $Z$ _ant2 $=58.5+j * 34.4$

ㅁeasurement @ $Z$ _ant3 $=81.2+j * 19.3$

- PA Model

FIGURE 19: Gain compression (AMAM) versus input power for three calculated impedances $\left(Z_{\_}\right.$ant $1=57.1+j * 20.1, Z_{\_}$ant $2=$ $58.5+j * 34.4$ and $Z_{-}$ant $\left.3=81.2+j * 19.3\right)$ using the synthesis procedure. PA model (lines) compared to load pull measurement (symbols). $F_{0}=8.2 \mathrm{GHz}$.

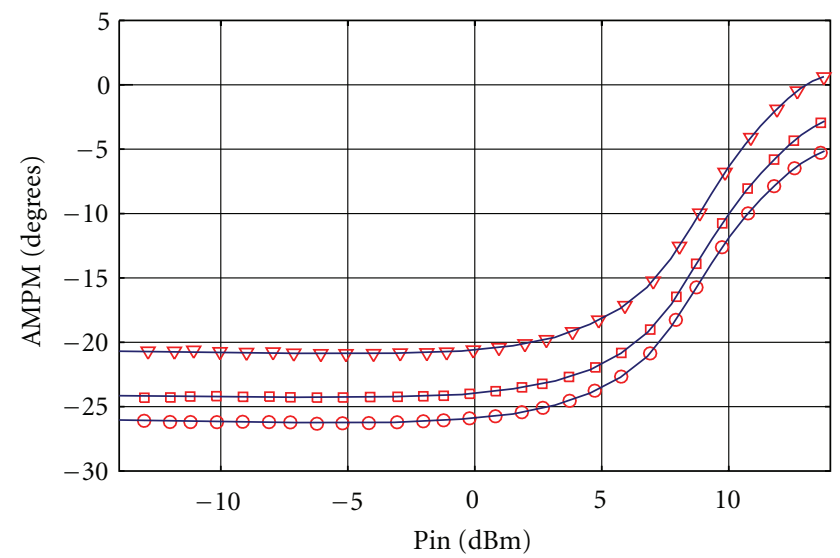

$\nabla \quad$ Measurement @ $Z \_$ant $1=57.1+j * 20.1$

○ Measurement @ $Z$ _ant2 $=58.5+j * 34.4$

Measurement @ $Z \_a n t 3=81.2+j * 19.3$ - PA Model

Figure 20: Phase modulation (AMPM) versus input power for three calculated impedances $\left(Z_{-}\right.$ant $1=57.1+j * 20.1, Z_{-}$ant $2=$ $58.5+j * 34.4$, and $Z_{-}$ant3 $\left.=81.2+j * 19.3\right)$ using the EM analysis tool. PA model (lines) compared to load pull measurement (symbols). $F_{0}=8.2 \mathrm{GHz}$.

very interesting. In fact, if couplings are taken into account through the synthesis procedure, it is possible to obtain an optimized radiation pattern and a reflected power wave $\left(b_{i}\right)$ almost equal to zero, as explained earlier in Section 2. In that case, the array and PA functioning are not disturbed. It is thus possible to eliminate isolators between PAs and antennas in TX chains, leading to a significant weight and cost reduction, added to lower dissipation losses.
TABLE 4: Calculated impedances for each antenna in the array $1 \times 4$ $F_{0}=8.2 \mathrm{GHz}\left(\theta=0^{\circ}, \theta=-10^{\circ}\right.$, and $\left.\theta=15^{\circ}\right)$.

\begin{tabular}{lccc}
\hline $\begin{array}{l}\text { Pointing } \\
\text { Angle }\end{array}$ & Probes & $Z_{E i}\left(Z_{\text {ant }}\right)$ & VSWR \\
\hline \multirow{3}{*}{$0^{\circ}$} & 1 & $58.5+j * 34.4$ & 1.9 \\
& 2 & $57.5+j * 19.5$ & 1.47 \\
& 3 & $56.9+j * 20.1$ & 1.48 \\
$-10^{\circ}$ & 4 & $62.4+j * 33.3$ & 1.87 \\
& 1 & $75.3+j * 23.6$ & 1.75 \\
& 2 & $57.2+j * 18.8$ & 1.45 \\
& 3 & $57.1+j * 20.1$ & 1.49 \\
$+15^{\circ}$ & 4 & $47.7+j * 25.6$ & 1.68 \\
& 1 & $43.8+j * 26.1$ & 1.76 \\
& 2 & $55.6+j * 18.9$ & 1.45 \\
& 3 & $58+j * 17.7$ & 1.43 \\
& 4 & $81.2+j * 19.3$ & 1.76 \\
\hline
\end{tabular}

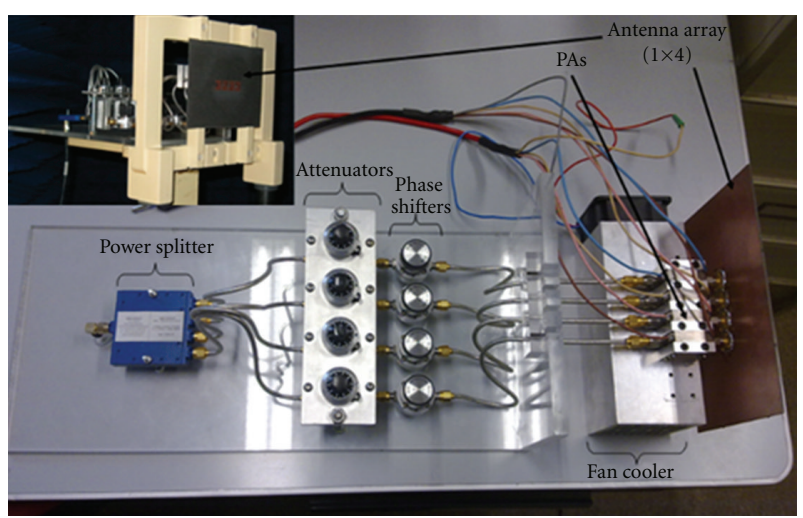

Figure 21: Phase Active antenna demonstrator.

As previously mentioned, the idea is to correct signal, that is, magnitude and phase, at each PA input in order to ensure both the desired weights at each antenna access and a null reflected wave. AMAM $(\delta|a|)$ and AMPM $(\delta \phi)$ values are obtained from PAs model response in terms of AMAM and AMPM at an available input power range $\left(P_{\text {in }}\right)$. The desired weights $\left(\left|a_{i}\right| e^{j \varphi i}\right)$ which were calculated by the synthesis procedure can be established by feeding each PA by a corrected magnitude $(|a|-\delta|a|)$ and phase $(\varphi-\delta \varphi)$.

Figures 22 and 23 compare the measured radiation patterns with PA (active antenna demonstrator), the measured radiation patterns without PA (synthesis procedure validation), and simulated radiation patterns without PA obtained through the synthesis procedure for a $-10^{\circ}$ and $+15^{\circ}$ pointing angle. The measured radiation patterns without PA were achieved by feeding each antenna with the calculated weights $a_{i}$ and loaded with corresponding matching impedance as well.

Figures 22 and 23 show a good agreement between the main lobes for the measured results (with and without PA) and simulated ones.

Some minor differences on the side lobe levels appear which are likely caused by the asymmetry of feeding antennas 


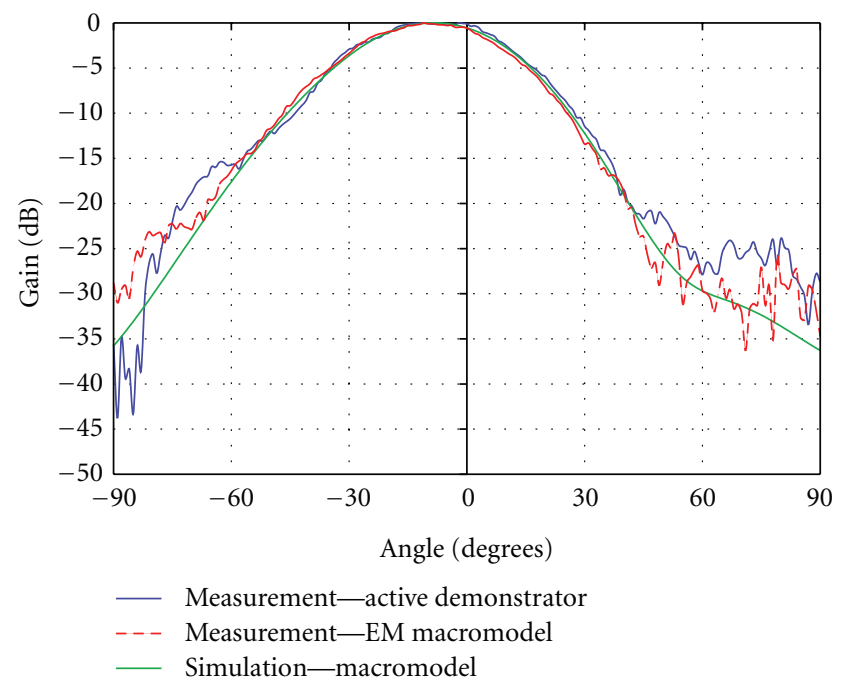

FIGURE 22: Radiation pattern comparison $\left(-10^{\circ}\right)$. Active demonstrator measurement (solid line). Measurement without PA (big dashed line). EM macro-model simulation (small dashed line). $F_{0}=8.2 \mathrm{GHz}$.

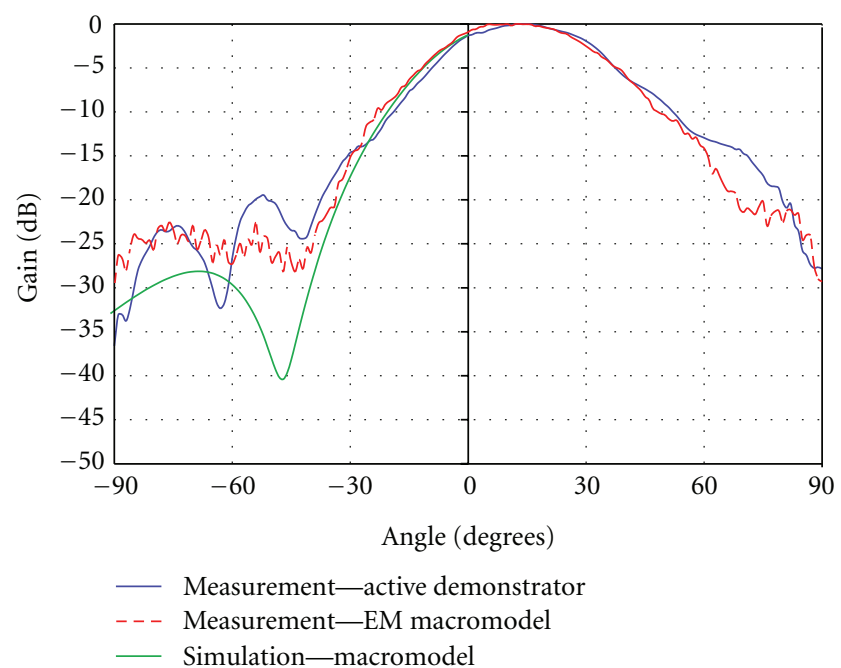

Figure 23: Radiation pattern comparison $\left(+15^{\circ}\right)$. Active demonstrator measurement (solid line). Measurement without PA (big dashed line). EM macromodel simulation (small dashed line). $F_{0}=8.2 \mathrm{GHz}$.

probes. Another reason for these differences may come from the inaccuracies during the measuring procedure.

The synthesis procedure of Section 2 has been successfully applied to the prediction of each matching impedance of array antennas that can be linked to a PA model. The results take into account large output mismatches, allowing to define the loading impedances of active circuits (PAs) and to optimize the overall performances. Measurements for different radiation patterns, with or without PA, demonstrate the robustness and accuracy of the mixed simulation concept and offer new perspectives for active electronically scanned array (AESA) applications with the elimination of isolators from a TX chain.

\section{Synthesis of Parasitic Antenna Arrays}

The synthesis procedure of Section 2 has been applied to antenna array, either in passive or active architecture. However, multielement antennas also include parasitic antenna array. The main interest of such design is to avoid a feed distribution network and its associated cost and losses. Therefore, only one element is driven, the others are electromagnetically coupled to obtain the objective pattern.

One of the well-known designs with parasitic elements is the Yagi-Uda. This antenna has been widely derived in printed designs $[27,28]$. Another important field of interest for parasitic array is reconfigurable antennas [29]. A lot of designs have been developed for mobile antennas and MIMO applications, especially by including diodes or micro electro mechanical systems (MEMS) [30]. However, these antennas are most often conceived with parametric studies because of the strong couplings which are difficult to predict and to include in the modeling process. As a whole, larger structures require too much time computing whereas they can of interest for some applications mentioned in Section 1. In this part, we solve this problematic with the previous synthesis method. In order to be used, it has been fitted to multielement antenna with a single driven element. It is then 
applied to a Yagi-Uda printed design and to a reflectarray. In fact, this last category of antenna is based on a single feed excitation which is electromagnetically coupled to an array of radiating elements. These ones can be assimilated as parasitic elements fed by specific weights depending on the impinging waves coupled with the cells.

4.1. Synthesis of Parasitic Arrays. Let us consider the parasitic antenna array made of $n$ elementary antennas. One antenna is fed and the others are connected to reactive loads. For this kind of design, the aim is to synthesize the loads that will satisfy the radiation pattern. Looking at Figure 24, the resulting radiation pattern is a linear combination of all the antenna radiation patterns, as mentioned in (33) where $a_{1 \ldots n}$ are complex weights, $\Phi_{1 \ldots n}$ are the radiation patterns of the $n$ antennas:

$$
\Phi=a_{1} \cdot \Phi_{1}+a_{2} \cdot \Phi_{2} \cdots+a_{g} \cdot \Phi_{g} \cdots+a_{n} \cdot \Phi_{n} .
$$

Only the antenna named " $g$ " is fed through its port, the others are excited by mutual coupling. A set of reactive loads $X_{i}$ will be chosen to control the complex weights $a_{i}$. The multiple-antenna device is characterized by a $S_{n \times n}$ matrix. In order to facilitate the resolution, this $S_{n x n}$ is written using three blocks (34):

$$
S_{n \times n}=\left[\begin{array}{c}
S 1_{(g-1) \times n} \\
S 2_{1 \times n} \\
S 3_{(n-g) \times n}
\end{array}\right] .
$$

with,

$$
\begin{aligned}
S 1_{(g-1) \times n} & =\left[\begin{array}{ccc}
S_{1,1} & \cdots & S_{1, n} \\
\vdots & \ddots & \vdots \\
S_{g-1,1} & \cdots & S_{g-1, n}
\end{array}\right], \\
S 2_{1 \times n} & =\left[\begin{array}{ccc}
S_{g, 1} & \cdots & S_{g, n}
\end{array}\right], \\
S 3_{(n-g) \times n} & =\left[\begin{array}{ccc}
S_{g+1,1} & \cdots & S_{g+1, n} \\
\vdots & \ddots & \vdots \\
S_{n, 1} & \cdots & S_{n, n}
\end{array}\right] .
\end{aligned}
$$

The complex weights are similarly decomposed (36):

$$
\begin{gathered}
(a)=\left(\begin{array}{c}
A 1_{(g-1) \times 1} \\
A 2_{1 \times 1} \\
A 3_{(n-g) \times 1}
\end{array}\right), \\
A 1_{(g-1) \times 1}=\left(\begin{array}{c}
a_{1} \\
\vdots \\
a_{g-1}
\end{array}\right), \quad A 2_{1 \times 1}=a_{g}, \quad A 3_{(n-g) \times 1}=\left(\begin{array}{c}
a_{g+1} \\
\vdots \\
a_{n}
\end{array}\right) .
\end{gathered}
$$

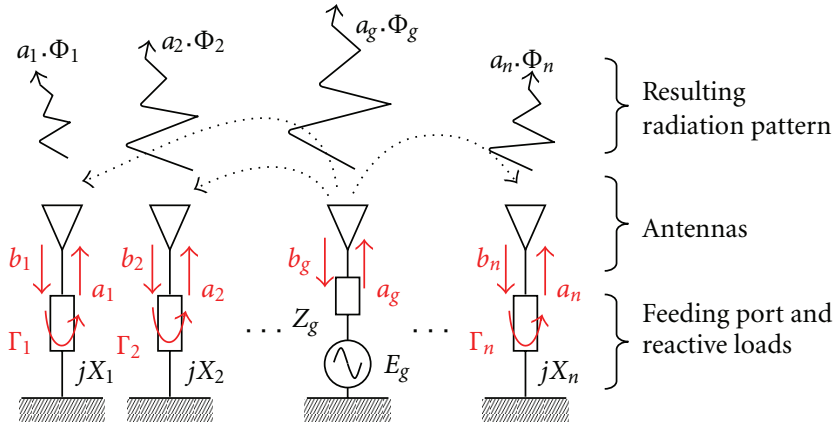

FIgURE 24: Principle of a parasitic antenna array.

For the mathematical formulation, the reactive loads connected to the antennas are changed into reflection coefficients (38):

$$
\Gamma_{i}=\frac{b_{i}}{a_{i}}=\frac{z_{0}-j X_{i}}{z_{0}+j X_{i}}
$$

where $z_{0}$ is the port impedance.

Let us define the relation (39):

$$
\left(\begin{array}{l}
A 1 \\
A 3
\end{array}\right)=\text { GAMMA } \cdot\left[\begin{array}{l}
S 1 \\
S 3
\end{array}\right] \cdot\left(\begin{array}{l}
A 1 \\
A 2 \\
A 3
\end{array}\right) .
$$

With

$$
\begin{aligned}
& \operatorname{GAMMA}_{(n-1) \times(n-1)} \\
& \quad=\cdots \operatorname{diag}\left(\Gamma_{1}, \Gamma_{2}, \ldots \Gamma_{g-1}, \Gamma_{g+1}, \ldots \Gamma_{n}\right) .
\end{aligned}
$$

The problem is now to find the reflection coefficients $\left(\Gamma_{i}\right)$ and the complex weights ( $A 1$ and $A 3)$ able to satisfy the radiation objective.

A simplified approach (which is efficient but not systematically the optimum choice) consists in fixing the phase of each complex weight in order to build a constructive summation in a steering direction. We can set these phases using the relation (41):

$$
\begin{aligned}
\angle a_{i}=\Delta_{i}= & \angle a_{g}+\cdots \\
& \angle \Phi_{g}\left(\theta_{\text {steer }}, \varphi_{\text {steer }}\right)-\angle \Phi_{i}\left(\theta_{\text {steer }}, \varphi_{\text {steer }}\right) .
\end{aligned}
$$

Since GAMMA is a diagonal matrix, it can be written:

$$
\left\|\begin{array}{l}
A 1 \\
A 3
\end{array}\right\|=\| \text { GAMMA }\|\cdot\|\left[\begin{array}{l}
S 1 \\
S 3
\end{array}\right] \cdot\left(\begin{array}{l}
A 1 \\
A 2 \\
A 3
\end{array}\right) \| .
$$

If $\left\|\Gamma_{i}\right\| \approx 1$, (42) can be simplified, resulting in (43):

$$
\left\|\begin{array}{l}
A 1 \\
A 3
\end{array}\right\|=\left\|\left[\begin{array}{l}
\Delta 1 \\
\Delta 3
\end{array}\right] \cdot\right\| \begin{aligned}
& A 1 \\
& A 2 \\
& A 3
\end{aligned}\|\| .
$$


with

$$
\left[\begin{array}{l}
\Delta 1 \\
\Delta 3
\end{array}\right]=\left[\begin{array}{l}
S 1 \\
S 3
\end{array}\right] * \operatorname{diag}\left(e^{j \angle a_{i}}\right)
$$

$\|A 1\|$ and $\|A 3\|$ are the unknowns to define. Equation (43) can be quickly solved using a convergence algorithm.

Once $\|A 1\|$ and $\|A 3\|$ have been found, the associated reactive loads can be easily deduced from the reflection coefficients with (45):

$$
\mathrm{GAMMA}=\left(\begin{array}{l}
\mathrm{A} 1 \\
\mathrm{~A} 3
\end{array}\right) \cdot /\left(\left[\begin{array}{l}
\mathrm{S} 1 \\
\mathrm{~S} 3
\end{array}\right] \cdot\left(\begin{array}{l}
\mathrm{A} 1 \\
\mathrm{~A} 2 \\
\mathrm{~A} 3
\end{array}\right)\right) .
$$

The coefficients $\Gamma_{i}$ are the diagonal of the GAMMA matrix.

It becomes

$$
j X_{i}=z_{0} \frac{1+\Gamma_{i}}{1-\Gamma_{i}} .
$$

The last step is the determination of the input impedance for the parasitic antenna array configuration. Indeed, this impedance is a function of the interactions with the other antennas. It becomes

$$
Z_{E}=z_{0} \cdot \frac{a_{g}+b_{g}}{a_{g}-b_{g}}
$$

with $b_{g}=S 2 \cdot\left(\begin{array}{l}A 1 \\ A 2 \\ A 3\end{array}\right)$, and $A 2=a_{g}$.

The impedance matching is then achieved by changing $Z_{g}$ by $Z_{E}^{*}$.

\subsection{Application to the Design of a Yagi-Uda Antenna. With} the aforementioned relation, a straightforward design is proposed to illustrate the performances. The parasitic antenna array is a Yagi-Uda antenna composed of 15 identical dipoles printed on a $1.6 \mathrm{~mm}$ thickness FR4 substrate $\left(\varepsilon_{r}=4.3, \operatorname{tg} \delta=\right.$ 0.025 ). The dipole length is $40 \mathrm{~mm}$. The array is $270 \mathrm{~mm}$ long with a $15.63 \mathrm{~mm}$ step between each element. A ground plane is preserved at the rear of the substrate to receive the electronic parts. The operating frequency is $2.45 \mathrm{GHz}$, and the reactive loads are performed with surface-mounted devices (SMDs) components. The different dimensions are depicted in Figure 25.

4.2.1. Synthesis Description. The first step consists in the electromagnetic analysis. This analysis is performed with CST MWS. The numerical design is fitted with 15 ports lying in the middle of each dipole. The 15 dipoles are successively fed in order to build the $[S]$ matrix and to calculate the 15 radiation patterns. The obtained dataset is then used as inputs for (33) and (34).

The relations (33) to (47) are solved with MaTLAB in order to establish the best configuration, that is, feeding (or active) port provides the best performances in terms of directivity, impedance matching, and backward radiation.

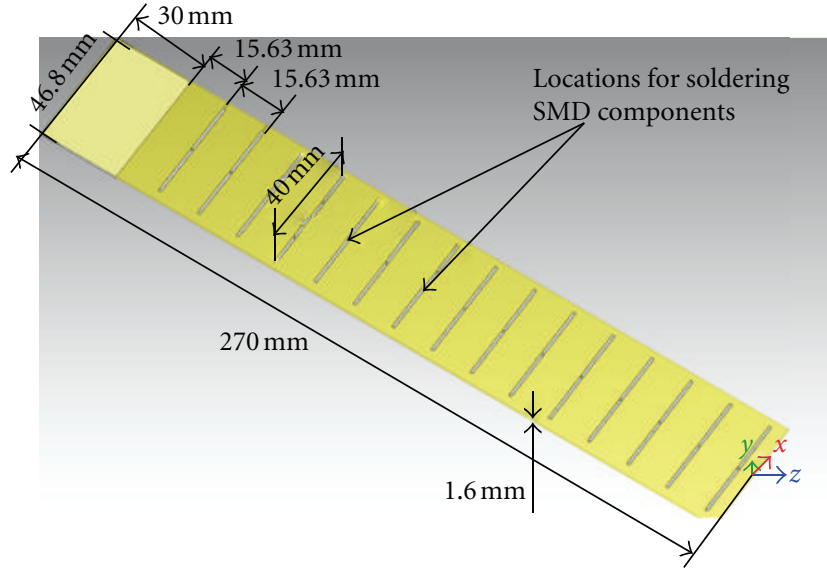

Figure 25: The printed Yagi-Uda antenna is made of 15 dipoles printed on a FR4 substrate.

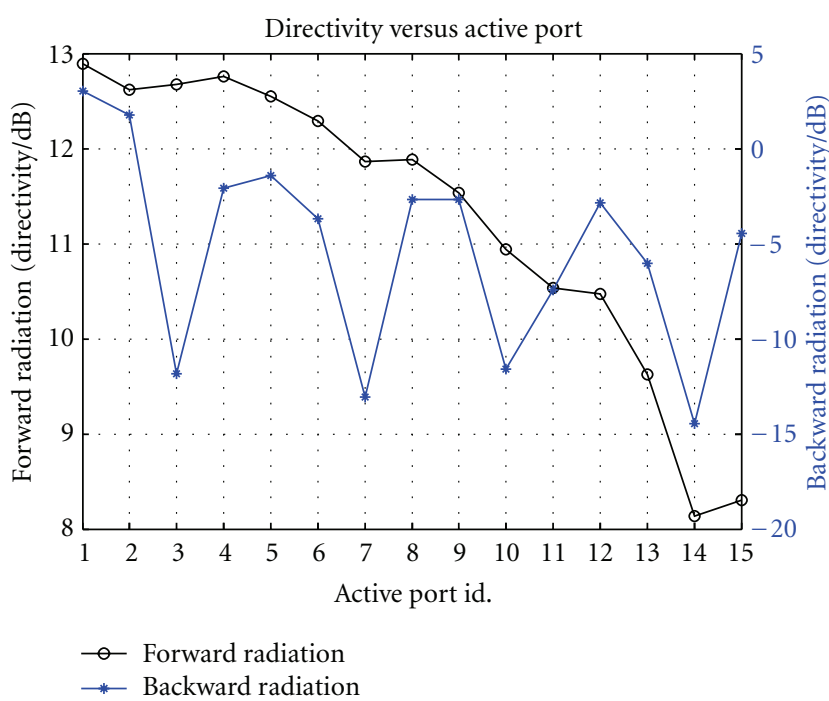

FiguRE 26: Forward and backward directivity as a function of the fed dipole.

The resulting 3D radiation patterns are simultaneously computed, as the antenna impedance when the reactive loads are connected.

Figure 26 shows the directivity and the backward radiation versus the feeding port index. The set of reactive loads is changing with the feeding port. This Figure is useful to select the most appropriate dipole to use as a feed among the 15 .

The Figure 27 shows the input impedance as a function of the active port id.

Notice that whatever the fed dipole among the 15 elements, the input impedance remains steady and close to $Z e \approx 30+j$.

Given these characteristics, the third port has been chosen as driven element. The main argument justifying this choice is the front to backward radiation. The directivity is $12.67 \mathrm{~dB}$ in the forward direction against $-11 \mathrm{~dB}$ for the backward radiation.

The corresponding radiation pattern is presented in Figures 28 and 29. 
TABLE 5: Weightings for the coupled radiation patterns and reactive loads that are to connected to the non-fed dipoles.

\begin{tabular}{|c|c|c|c|c|}
\hline \multirow[t]{2}{*}{ Dipoles $i$} & \multirow{2}{*}{$a_{i}$ (weights for the coupled radiation patterns) } & \multicolumn{3}{|c|}{$\begin{array}{c}\text { Reactive loads }\left(j X_{i}\right) \text { and input impedance }\left(Z_{E}\right) \text { that } \\
\text { produce the beam steering at } 2.45 \mathrm{GHz}\end{array}$} \\
\hline & & $j X_{i} / Z_{E}$ & Inductances $L_{i}$ & Capacitors $C_{i}$ \\
\hline 1 (reflector) & $0.214 \cdot \exp ^{\left(j * 77^{\circ}\right)}$ & $j X_{1}=j * 49$ & $3.18 \mathrm{nH}$ & \\
\hline 2 (reflector) & $0.342 \cdot \exp ^{\left(j * 58.5^{\circ}\right)}$ & $j X_{2}=j * 59.8$ & $3.88 \mathrm{nH}$ & \\
\hline 3 (feeder) & 1 & $Z_{E}=26.7-j * 0.5$ & - & - \\
\hline 4 (director) & $0.382 \cdot \exp ^{\left(-j * 64.5^{\circ}\right)}$ & $j X_{4}=-j * 83.1$ & & $0.78 \mathrm{pF}$ \\
\hline 5 (director) & $0.329 \cdot \exp ^{\left(-j * 97.5^{\circ}\right)}$ & $j X_{5}=-j * 105$ & & $0.62 \mathrm{pF}$ \\
\hline 6 (director) & $0.276 \cdot \exp ^{\left(-j * 142^{\circ}\right)}$ & $j X_{6}=-j * 113$ & & $0.57 \mathrm{pF}$ \\
\hline 7 (director) & $0.249 \cdot \exp ^{\left(j * 156^{\circ}\right)}$ & $j X_{7}=-j * 107$ & & $0.61 \mathrm{pF}$ \\
\hline 8 (director) & $0.273 \cdot \exp ^{\left(j * 110^{\circ}\right)}$ & $j X_{8}=-j * 156$ & & $0.42 \mathrm{pF}$ \\
\hline 9 (director) & $0.274 \cdot \exp ^{\left(j * 66.3^{\circ}\right)}$ & $j X_{9}=-j * 143$ & & $0.45 \mathrm{pF}$ \\
\hline 10 (director) & $0.23 \cdot \exp ^{\left(j * 10.6^{\circ}\right)}$ & $j X_{10}=-j * 117$ & & $0.55 \mathrm{pF}$ \\
\hline 11 (director) & $0.233 \cdot \exp ^{\left(-j * 44.7^{\circ}\right)}$ & $j X_{11}=-j * 167$ & & $0.39 \mathrm{pF}$ \\
\hline 12 (director) & $0.273 \cdot \exp ^{\left(-j * 93.6^{\circ}\right)}$ & $j X_{12}=-j * 129$ & & $0.50 \mathrm{pF}$ \\
\hline 13 (director) & $0.244 \cdot \exp ^{\left(-j * 148^{\circ}\right)}$ & $j X_{13}=-j * 78.9$ & & $0.82 \mathrm{pF}$ \\
\hline 14 (director) & $0.191 \cdot \exp ^{\left(j * 148^{\circ}\right)}$ & $j X_{14}=-j * 107$ & & $0.61 \mathrm{pF}$ \\
\hline 15 (director) & $0.292 \cdot \exp ^{\left(j * 78.9^{\circ}\right)}$ & $j X_{15}=-j * 71.1$ & & $0.91 \mathrm{pF}$ \\
\hline
\end{tabular}

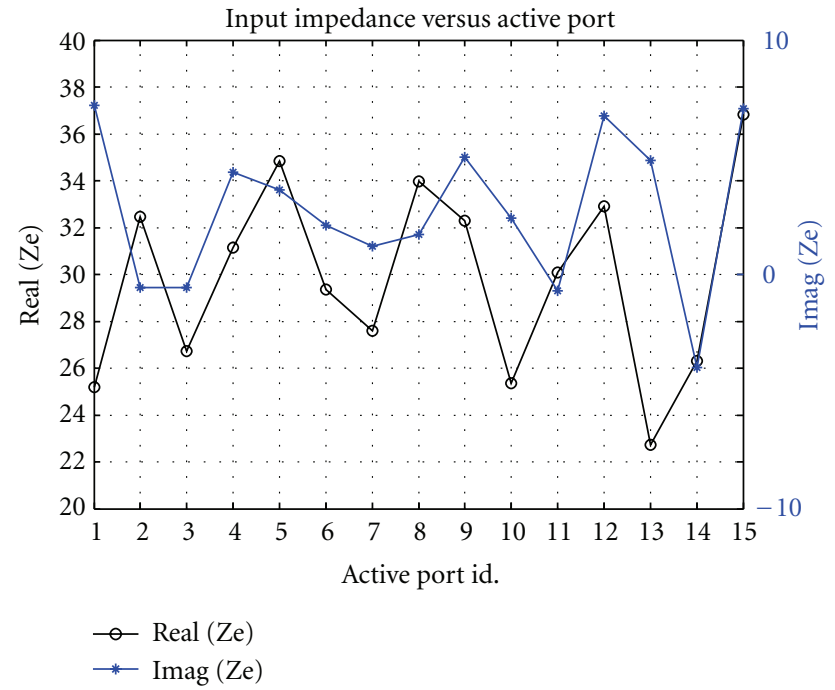

FIGURE 27: Input impedance versus the feeding port index (port id.).

The reactive loads optimizing the radiation pattern are given in the third column in Table 5.

The second column indicates the weights $a_{i}$ corresponding to the contribution of each dipole. The fourth and the fifth columns give the lumped elements required to synthesise the reactive loads of the third column.

4.2.2. Finalizing the Design and Characterization. The active dipole is connected to an SMA connector at the rear of the PCB with a coplanar waveguide. A balun optimises the matching impedance and performs a symmetric supply. In order to facilitate the link with the coplanar waveguide, the

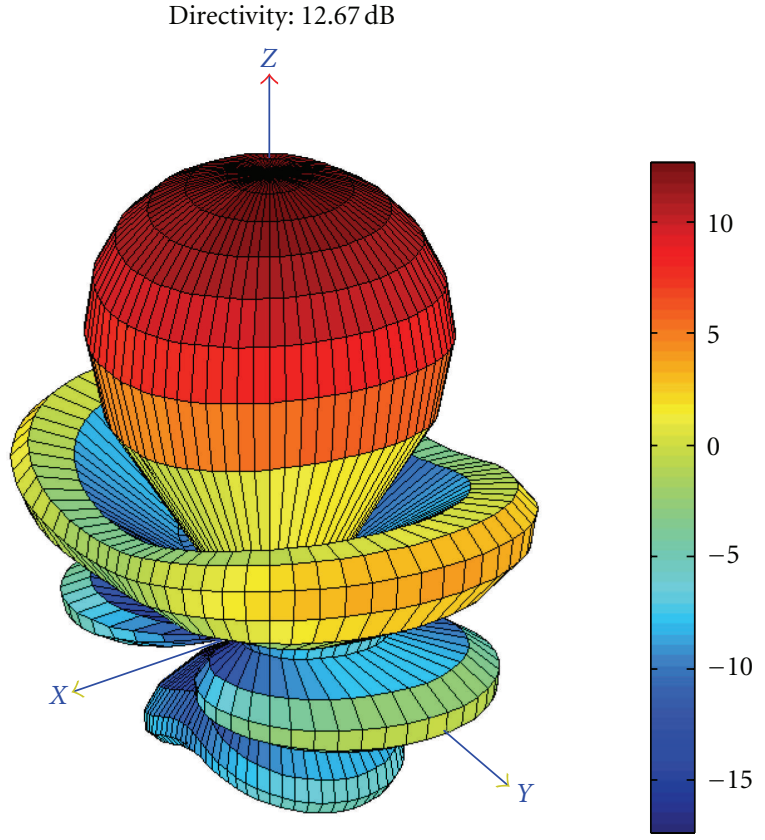

FIgURE 28: The radiation pattern is computed with (33). The complex weights and the reactive loads are reported in Table 5. The directivity is $12.67 \mathrm{~dB}$.

active dipole, the first and second dipoles, and their reactive SMD components are moved to the back of the substrate as can be seen in Figure 30. It shows the Yagi-Uda antenna with the reactive loads. The CST MWS full-wave simulation uses the realistic SMD components values (including loss resistance) provided by Coilcraft for the inductors and AVX for the capacitors. The selected components are listed in Table 6. They can be compared to the theoretical objectives of Table 5 . 


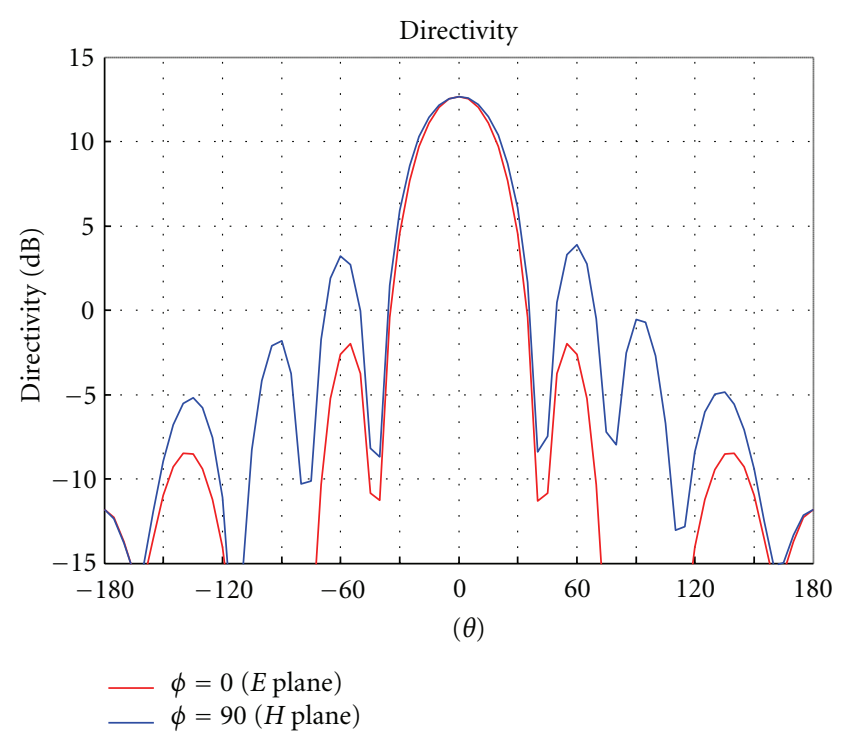

Figure 29: $E$ - and $H$-plane cuts of the radiation pattern at $2.45 \mathrm{GHz}$.

TABLE 6: The objective lumped elements are replaced by in-stock values.

\begin{tabular}{lcc}
\hline Dipole $i$ & $\begin{array}{c}\text { Purchased inductances } \\
\text { (Coilcraft) }(\mathrm{nH})\end{array}$ & $\begin{array}{c}\text { Purchased capacities } \\
(\mathrm{AVX})(\mathrm{pF})\end{array}$ \\
\hline 1 & 3.3 & \\
2 & 3.9 & 0.8 \\
4 & & 0.5 \\
5 & & 0.5 \\
6 & & 0.5 \\
7 & & 0.5 \\
8 & 0.5 \\
9 & 0.5 \\
10 & 0.5 \\
11 & 0.5 \\
12 & 0.8 \\
13 & 0.5 \\
14 & 0.9 \\
\hline
\end{tabular}

The antenna including the coplanar waveguide, the balun, and the realistic lumped elements has been simulated. All lumped elements have a $0.1 \mathrm{Ohm}$ serie resistance. The transient computation predicts $11.6 \mathrm{~dB}$ directivity. Figure 31 shows that the radiation pattern is not much affected by the matching circuit.

Looking at Figures 29 and 32, we can conclude that the backward radiation is greater with the balun. Nevertheless, the performance satisfies our requirements. The antenna manufactured is presented in Figure 33. The measured return loss is presented in Figure 34 and compared with the simulation. The experimental result agrees well the prediction, validating the synthesis procedure and the SMD components choice.

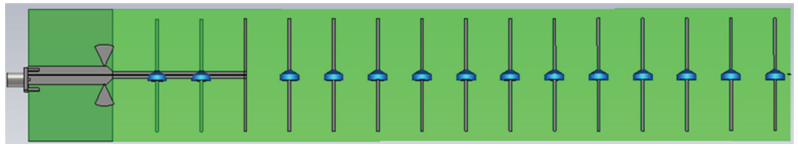

FIgURE 30: The active dipole is fed through a coplanar waveguide. A balun achieves both the matching impedance and the symmetrisation of the currents into the coplanar waveguide. Blue parts correspond to the lumped elements.

The main interest of this design consists in the straightforward design without full-wave and time-consuming parametric studies which are classically used for parasitic antenna. Moreover, from the manufacturing process point of view, such an antenna can be realized as a generic one with only a few dataset of SMD components to satisfy a radiation objective. This concept of antenna might be useful when a low-cost design is required. Notice that a very attractive and original point of the synthesis procedure presented here is the possibility to define the best element to be driven in the parasitic array. With parametric design, such a study would have led to a lot of combination and time consuming. For the antenna presented, once the scattering matrix and the radiation patterns have been computed with the full-wave simulation, then it takes only a few seconds to find the best combination of reactive loads and driven element.

4.3. Focus on a Particular Parasitic Array: The Reflectarray Antenna. The reflectarray antenna can be investigated as a parasitic antenna array. The only difference is that the active port is outside and far from the array. This feed illuminates the cell-array which gathers the EM energy and restitutes this energy with the usual behavior of an array antenna. Looking at Section 4.1, the problem is similar. It can be reduced to consider only the reflective panel (built with periodic cells including ports) if each port is submitted to an initial perturbation linked to the external feed. Each cell acts as an elementary antenna in an array.

In the following developments, the primary feed does not interact with the cells of the panel, it injects an initial excitation $b 0_{i}$, as depicted on Figure 35.

The panel is characterized by a scattering matrix $S_{n x n}$ and the radiation pattern of each cell. The incident powers $b 0_{i}$ depend on the radiation of the external feed.

The diffraction of the reactive panel is driven by the relation (48):

$$
a_{i}=\Gamma_{i} \cdot\left(b_{i}+b 0_{i}\right) .
$$

Including the couplings, we obtain

$$
\left[I_{n \times n}-\operatorname{diag}(\Gamma)_{n \times n} \cdot S_{n \times n}\right]\left(a_{i}\right)=\operatorname{diag}(\Gamma)_{n \times n} \cdot\left(b 0_{i}\right) .
$$

This relation can be solved as in the previous section:

$$
\operatorname{diag}\left(\Gamma^{-1}\right)_{n \times n} \cdot\left(a_{i}\right)=S_{n \times n} \cdot\left(a_{i}\right)+\left(b 0_{i}\right) .
$$

Let us consider that $\left\|\Gamma_{i}\right\| \approx 1$ be comes

$$
\left\|\left(a_{i}\right)\right\|=\left\|\Delta_{n \times n} \cdot\right\|\left(a_{i}\right)\left\|+\left(b 0_{i}\right)\right\| .
$$




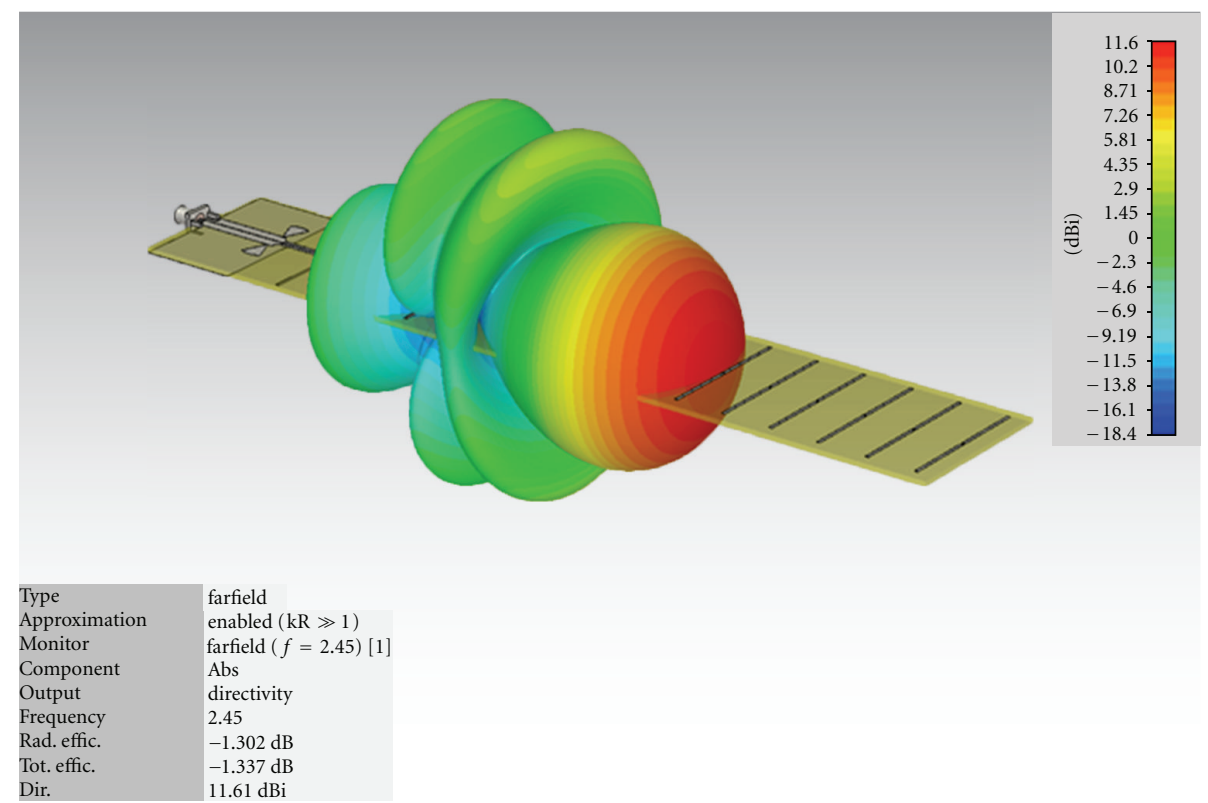

FIGURE 31: Radiation pattern computed with a full wave simulation (CST) at $2.45 \mathrm{GHz}$. The directivity is $11.6 \mathrm{~dB}$.

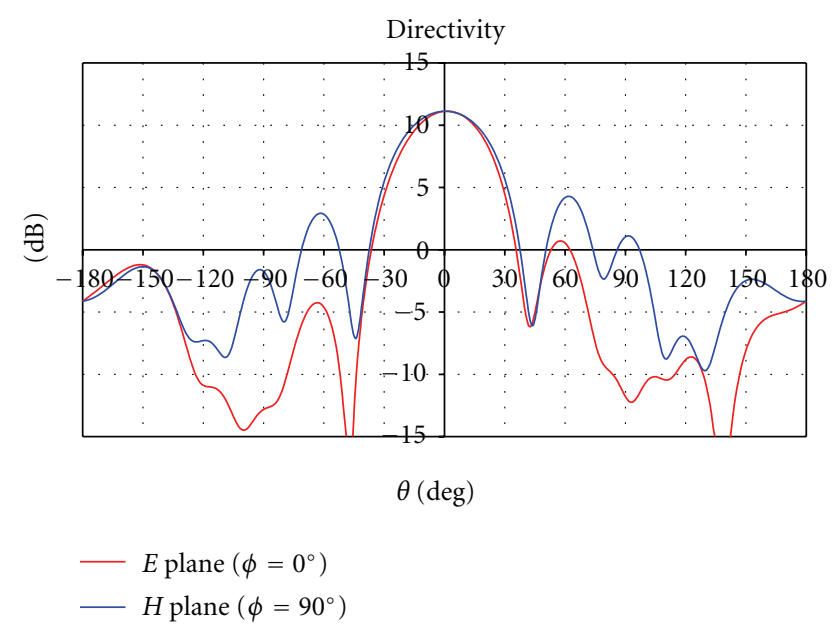

FIGURE 32: $E$ - and $H$-plane cuts of the radiation pattern at $2.45 \mathrm{GHz}$ including realistic values of the SMD components. The realized gain (simulated) reaches $9.74 \mathrm{~dB}$ (substrate and SMD ohmic losses).

with

$$
\Delta_{n \times n}=S_{n \times n} \cdot \operatorname{diag}\left(e^{j \angle a_{i}}\right) .
$$

This supposes that the phase distribution of the weighting is a sufficient condition to focus the beam (41):

$$
\operatorname{diag}(\Gamma)_{n \times n}=\left(a_{i}\right) \cdot /\left(\left(b 0_{i}\right)+S_{n \times n} \cdot\left(a_{i}\right)\right) .
$$

The reactive loads are given by (46).

One of the most interesting comments on this demonstration is linked to relations (51) and (52). In fact, to obtain the desired radiation pattern, the phase distribution is defined provided an offset phase reference which appears in (52). Notice that this offset has also a direct impact on the

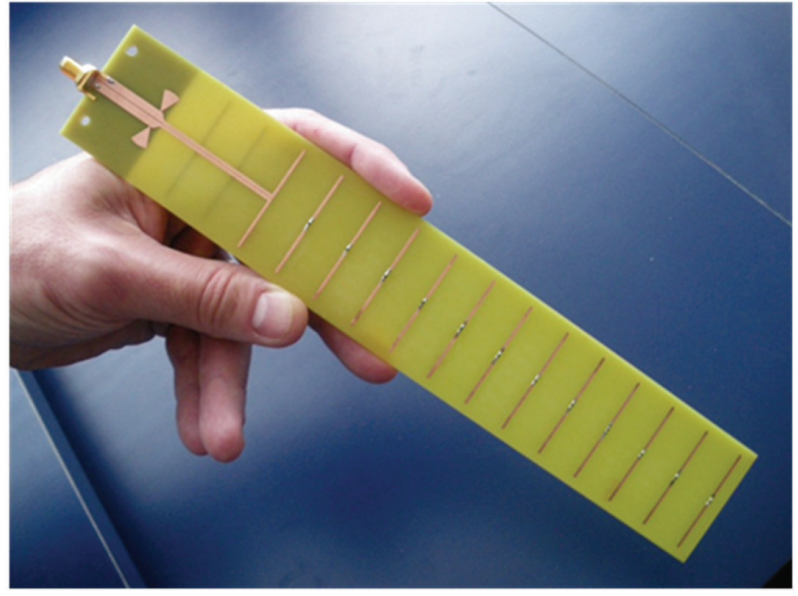

FIgure 33: Prototype of the Yagi-Uda antenna including the SMD inductors and capacitors.

magnitude distribution as demonstrated in (51). As a whole, the offset phase reference is a degree of freedom to improve the performances of the reflectarray.

\section{Conclusion}

In this article, a simple and multipurpose synthesis procedure for the design of multielement antenna has been described. It is based on a simple formulation including couplings. The main objective was to describe some antenna architectures or concepts where the couplings cannot be avoided. The different solutions developed rely on the accurate definition of the constraints on the circuits (feed distribution network, PA, reactive loads, etc.) connected to the antenna in order to perform an efficient combined design. The procedure has been successfully applied to 


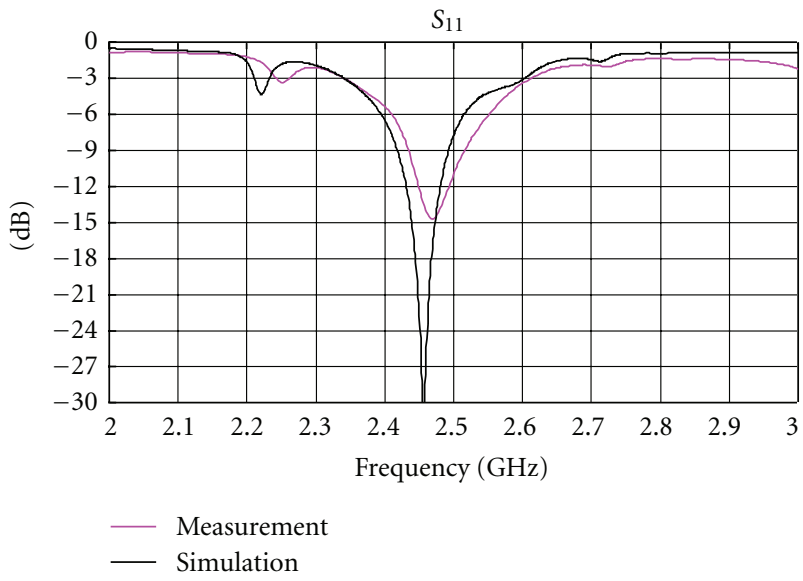

FIGURE 34: Comparison of the measured and simulated return loss.

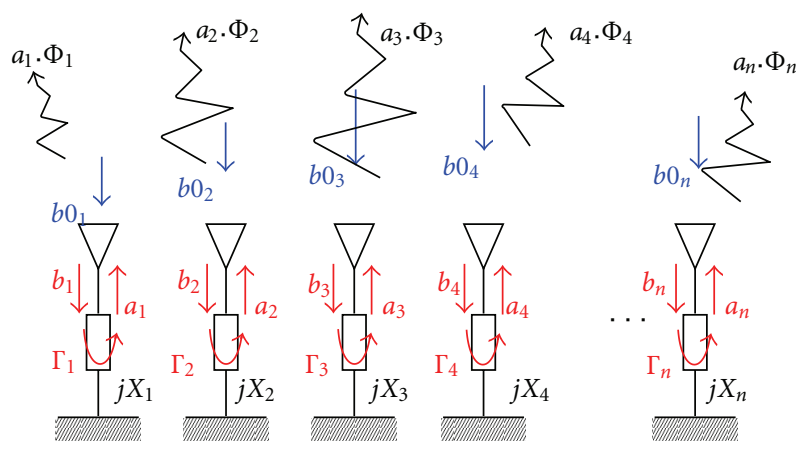

FIgURE 35: The illumination of the reactive panel by the external feed injects $b 0_{i}$ in each cell.

different categories of antennas of limited size, which is an additional constraint because of the severe interactions with the edge and antenna neighborhood. The synthesis procedure has been validated by cosimulation results and by measurements in order to guarantee its efficiency.

At first, passive antenna arrays have been conceived and measured with different objectives on the radiation pattern or on axial ratio. The interest of a synthesis including couplings has also been demonstrated on active array where the performances of amplifiers have been well predicted. An opportunity to eliminate isolators on TX chains has been proved.

Then, the case of parasitic antenna arrays has been investigated by fitting the method to a single driven element. Outside the synthesis performances, it has allowed an accurate study of these antennas, especially in the choice of the driven element and the prediction of matching and radiation performances. Notice that, thanks to this synthesis procedure, a straightforward design is possible without parametric simulation, leading to a significant time gain and improved performances. A specific investigation on reflectarray antennas has also been performed with this procedure. It has been shown that such architectures can be studied as parasitic antenna arrays in order to accurately define their performances. This is an important fact to notice because this kind of antennas often suffers from modeling accuracy problems, leading to a limited distribution in the telecommunications market.

\section{References}

[1] R. J. Mailloux, "Electronically scanned array," in Synthesis Lecture on Antennas, p. 82, Morgan \& Claypool Publishers, 2007.

[2] K. S. Rao and H. J. Moody, "Modeling of shaped beam satellite antenna patterns," IEEE Transactions on Antennas and Propagation, vol. 35, no. 6, pp. 632-642, 1987.

[3] C. A. Balanis, Antenna Theory Analysis and Design, Wiley Interscience, 3rd edition, 2005.

[4] D. M. Pozar, "Active element pattern," IEEE Transactions on Antennas and Propagation, vol. 42, no. 8, pp. 1176-1178, 1994.

[5] S. Zhang, S. Gong, Q. Gong, Y. Guan, and B. Lu, "Application of the active element pattern method for calculation of the scattering pattern of large finite arrays," IEEE Antennas and Wireless Propagation Letters, vol. 10, pp. 83-86, 2011.

[6] I. J. Gupta and A. A. Ksienski, "Effect of mutual coupling on the performance of adaptative arrays," IEEE Transactions on Antennas and Propagation, vol. 31, no. 5, pp. 785-791, 1983.

[7] H. T. Hui, "Improved compensation for the mutual coupling effect in a dipole array for direction finding," IEEE Transactions on Antennas and Propagation, vol. 51, no. 9, pp. 2498-2503, 2003.

[8] H. S. Lui, H. T. Hui, and M. S. Leong, "A note on the mutualcoupling problems in transmitting and receiving antenna arrays," IEEE Antennas and Propagation Magazine, vol. 51, no. 5, pp. 171-176, 2009.

[9] H. S. Lui and H. T. Hui, "Effective mutual coupling compensation for direction-of-arrival estimations using a new, accurate determination method for the receiving mutual impedance," Journal of Electromagnetic Waves and Applications, vol. 24, no. 2-3, pp. 271-281, 2010.

[10] H. T. Hui, "Decoupling methods for the mutual effect in antenna arrays: a review," Recent Patents on Engineering, vol. 1, pp. 187-193, 20047.

[11] R. Mittra, C. H. Chan, and T. Cwik, "Techniques for analyzing frequency selective surfaces-a review," Proceedings of the IEEE, vol. 76, no. 12, pp. 1593-1615, 1988.

[12] A. K. Bhattacharyya, Phased Array Antennas: Floquet Analysis, Synthesis, BFNs, and Active Array Systems, John Wiley \& Sons, Hoboken, NJ, USA, 2006.

[13] H. Aubert, "The concept of scale-changing network in the global electromagnetic simulation of complex structures," Progress In Electromagnetics Research B, no. 16, pp. 127-154, 2009.

[14] F. Khalil, A. Rashid, H. Aubert et al., "Application of scale changing technique - Grid computing to the electromagnetic simulation of reflectarrays," in Proceedings of the IEEE International Symposium on Antennas and Propagation and USNC/URSI National Radio Science Meeting, pp. 1-4, June 2009.

[15] R. Chiniard, A. Barka, and O. Pascal, "Fast and accurate modelling of large plane array antenna," in Proceedings of the 1st European Conference on Antennas and Propagation (EuCAP '06), pp. 1-6, 2006.

[16] C. C. Lu and W. C. Chew, "Multilevel algorithm for solving a boundary integral equation of wave scattering," Microwave and Optical Technology Letters, vol. 7, no. 10, pp. 466-470, 1994. 
[17] K. D’Ambrosio, R. Pirich, A. Kaufman, D. Mesecher, and P. Anumolu, "Parallel computation methods for enhanced MOM and MLFMM performance," in Proceedings of the IEEE Long Island Systems, Applications and Technology Conference, pp. 1-4, IEEE Long Island, May 2009.

[18] U. Jakobus and J. J. Van Tonder, "Challenges regarding the commercial implementation of the parallel MLFMM in FEKO," in Proceedings of the IEEE Antennas and Propagation Society International Symposium (APS '06), pp. 95-98, July 2006.

[19] J. Drouet, M. Thevenot, R. Chantalat et al., "Global synthesis method for the optimization of multifeed EBG antennas," International Journal of Antennas and Propagation, vol. 2008, Article ID 790358, 6 pages, 2008.

[20] T. Zhang and W. Ser, "Robust beampattern synthesis for antenna arrays with mutual coupling effect," IEEE Transactions on Antennas and Propagation, vol. 59, no. 8, pp. 2889-2895, 2011.

[21] A. El Sayed Ahmad, M. Thevenot, C. Menudier, M. Koubeissi, E. Arnaud, and T. Monédière, "Design of a coupled antenna array for mobile HyperLAN2 applications," International Journal of Microwave and Wireless Technologies, vol. 3, no. 6, pp. 609-614, 2011.

[22] E. W. C. Y. Lee, "Mutual coupling effect on maximum-ratio diversity combiners and application to mobile radio," IEEE Transactions on Communications, vol. 18, no. 6, pp. 779-791, 1970.

[23] T. Reveyrand, T. Gasseling, D. Barataud, S. Mons, and J.-M. Nébus, "A smart load-pull method to safely reach optimal matching impedances of power transistors," IEEE MTT-S Digest, pp. 1489-1492, 2007.

[24] G. Zakka El Nashef, F. Torres, S. Mons et al., "Power amplifiers modeling technique for reconfigurable antenna array application," in Proceedings of the Integrated Nonlinear Microwave and Millimetre-Wave Circuits (INMMiC '10), pp. 144-147, Göteborg, Sweden, 2010.

[25] G. Zakka El Nashef, F. Torres, S. Mons et al., "Second order extension of power amplifiers behavioral models for accuracy improvements," in Proceedings of the 40th European Microwave Conference (EuMW'10), pp. 1030-1033, Paris, France, 2010.

[26] G. Zakka El Nashef, F. Torres, A. El Sayed et al., "Development of an electromagnetic macro-model for reconfigurable array application," in Proceedings of the 4th European Conference on Antennas and Propagation (EuCAP '10), Barcelona, Spain, April 2010.

[27] Y. Qian, W. R. Deal, N. Kaneda, and T. Itoh, "Microstrip-fed quasi-Yagi antenna with broadband characteristics," Electronics Letters, vol. 34, no. 23, pp. 2194-2196, 1998.

[28] P. Y. Qin, A. R. Weily, Y. J. Guo, T. S. Bird, and C. H. Liang, "Frequency reconfigurable quasi-Yagi folded dipole antenna," IEEE Transactions on Antennas and Propagation, vol. 58, no. 8, pp. 2742-2747, 2010.

[29] J. T. Bernhard, "Reconfigurable antennas," in Synthesis Lectures on Antennas, Morgan and Claypool Publishers, 2007.

[30] L. Petit, L. Dussopt, and J. M. Laheurte, "MEMS-switched parasitic-antenna array for radiation pattern diversity," IEEE Transactions on Antennas and Propagation, vol. 54, no. 9, pp. 2624-2630, 2006. 

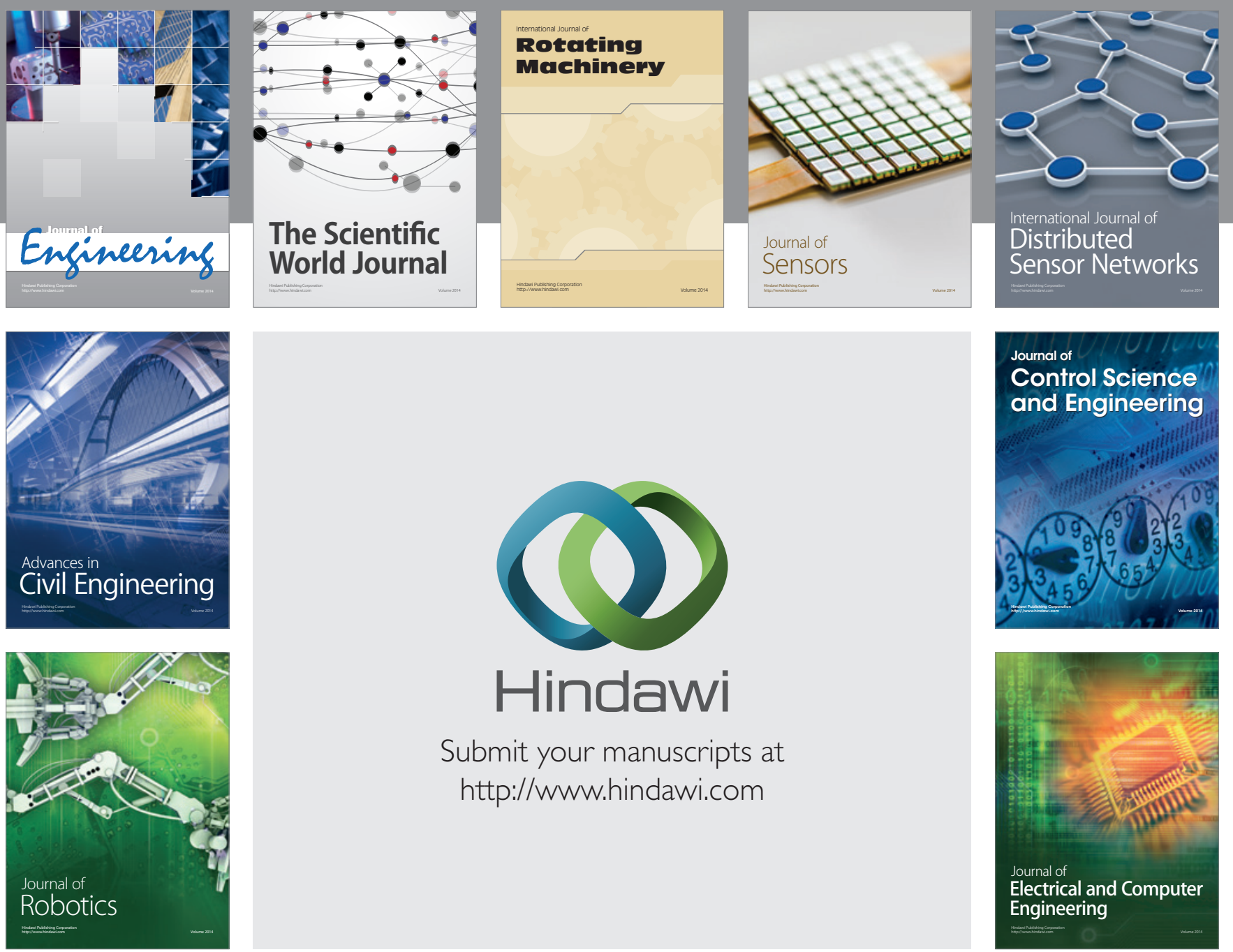

Submit your manuscripts at

http://www.hindawi.com
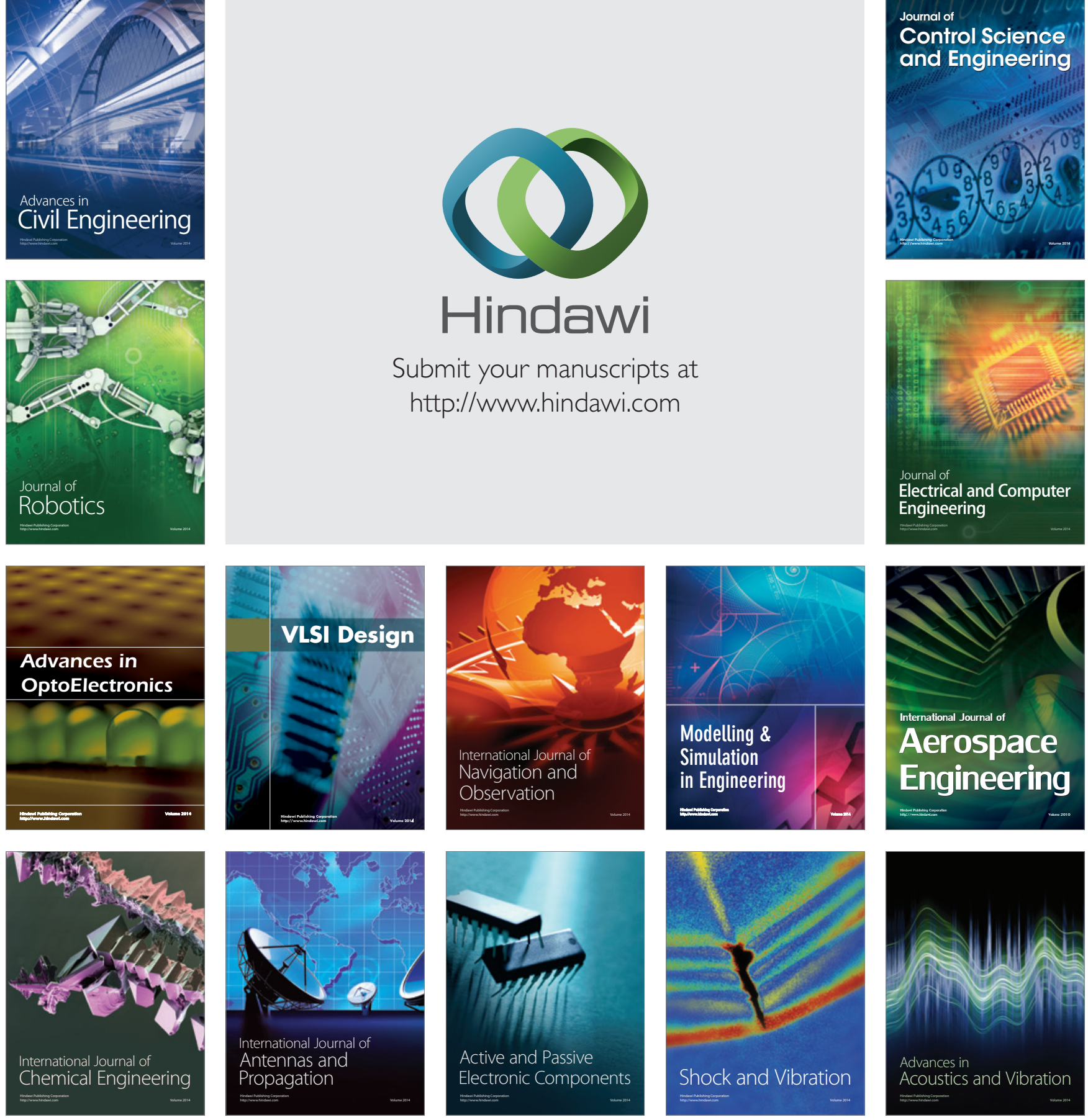